\title{
Generalized Lorentz Spaces and Applications
}

\author{
Hatem Mejjaoli \\ Department of Mathematics, College of Sciences, Taibah University, P.O. B 30002, Al Madinah Al Munawarah, Saudi Arabia
}

Correspondence should be addressed to Hatem Mejjaoli; hatem.mejjaoli@ipest.rnu.tn

Received 21 March 2013; Accepted 6 June 2013

Academic Editor: Jose Luis Sanchez

Copyright (C) 2013 Hatem Mejjaoli. This is an open access article distributed under the Creative Commons Attribution License, which permits unrestricted use, distribution, and reproduction in any medium, provided the original work is properly cited.

We define and study the Lorentz spaces associated with the Dunkl operators on $\mathbb{R}^{d}$. Furthermore, we obtain the Strichartz estimates for the Dunkl-Schrödinger equations under the generalized Lorentz norms. The Sobolev inequalities between the homogeneous Dunkl-Besov spaces and generalized Lorentz spaces are also considered.

"Dedicated to Khalifa Trimèche"

\section{Introduction}

Dunkl operators $T_{j}(j=1, \ldots, d)$ introduced by Dunkl in [1] are parameterized differential-difference operators on $\mathbb{R}^{d}$ that are related to finite reflection groups. Over the last years, much attention has been paid to these operators in various mathematical (and even physical) directions. In this prospect, Dunkl operators are naturally connected with certain Schrödinger operators for Calogero-Sutherlandtype quantum many-body systems [2-4]. Moreover, Dunkl operators allow generalizations of several analytic structures, such as Laplace operator, Fourier transform, heat semigroup, wave equations, and Schrödinger equations [5-11].

In the present paper, we intend to continue our study of generalized spaces of type Sobolev associated with Dunkl operators started in $[12,13]$. In this paper, we study the generalized Lorentz spaces, and we establish Sobolev inequalities between the homogeneous Dunkl-Besov spaces and many spaces as the homogeneous Dunkl-Riesz spaces and generalized Lorentz spaces.

As an application, we consider the Dunkl-Schrödinger equation

$$
\begin{gathered}
i \partial_{t} u+\triangle_{k} u=f(t, x), \quad(t, x) \in(0, \infty) \times \mathbb{R}^{d} \\
u_{\mid t=0}=g
\end{gathered}
$$

where $\triangle_{k}=\sum_{j=1}^{d} T_{j}^{2}$ is the Dunkl Laplace operator. We study the previous equation focusing on the following problems:

(1) Establish the Strichartz estimate under the generalized Lorentz norms.

(2) Illustrate applications to well posedness.

The contents of the paper are as follows. In Section 2, we recall some basic results about the harmonic analysis associated with the Dunkl operators. In Section 3, we introduce the homogeneous Dunkl-Besov spaces, the homogeneous Dunkl-Triebel-Lizorkin spaces, and the homogeneous Dunkl-Riesz potential spaces and we prove new embedding Sobolev theorem. In Section 4, we recall some facts about a real interpolation method. Next, we define the generalized Lorentz spaces and will pay special attention to the interpolation definition of these spaces. Section 5 is devoted to give a complete picture of the Sobolev type inequalities for the fractional Dunkl-Laplace operators. In Section 6, Strichartz estimates for the solution of the Dunkl-Schrödinger evolution equation are considered on a mixed normed space with generalized Lorentz norm with respect to the time variable. Finally, we establish Sobolev inequalities between the homogeneous Dunkl-Besov spaces and generalized Lorentz spaces, and we give many applications. 


\section{Preliminaries}

In order to confirm the basic and standard notations, we briefly overview the theory of Dunkl operators and related harmonic analysis. Main references are [1, 5, 6, 11, 14-17].

2.1. Root System, Reflection Group and Multiplicity Function. Let $\mathbb{R}^{d}$ be the Euclidean space equipped with a scalar product $\langle$,$\rangle , and let \|x\|=\sqrt{\langle x, x\rangle}$. For $\alpha$ in $\mathbb{R}^{d} \backslash\{0\}, \sigma_{\alpha}$ denotes the reflection in the hyperplane $H_{\alpha} \subset \mathbb{R}^{d}$ perpendicular to $\alpha$, that is, for $x \in \mathbb{R}^{d}, \sigma_{\alpha}(x)=x-2\|\alpha\|^{-2}\langle\alpha, x\rangle \alpha$. A finite set $R \subset \mathbb{R}^{d} \backslash\{0\}$ is called a root system if $R \cap \mathbb{R} \alpha=\{ \pm \alpha\}$ and $\sigma_{\alpha} R=R$ for all $\alpha \in R$. We normalize each $\alpha \in R$ as $\langle\alpha, \alpha\rangle=2$. We fix a $\beta \in \mathbb{R}^{d} \backslash \cup_{\alpha \in R} H_{\alpha}$ and define a positive root system $R_{+}$ of $R$ as $R_{+}=\{\alpha \in R \mid\langle\alpha, \beta\rangle>0\}$. The reflections $\sigma_{\alpha}, \alpha \in R$, generate a finite group $G \subset O(d)$, called the reflection group. A function $k: R \rightarrow \mathbb{C}$ on $R$ is called a multiplicity function if it is invariant under the action of $G$. We introduce the index $\gamma$ as

$$
\gamma=\gamma(k)=\sum_{\alpha \in R_{+}} k(\alpha)
$$

Throughout this paper, we will assume that $k(\alpha) \geq 0$ for all $\alpha \in R$. We denote by $\omega_{k}$ the weight function on $\mathbb{R}^{d}$ given by

$$
\omega_{k}(x)=\prod_{\alpha \in R_{+}}|\langle\alpha, x\rangle|^{2 k(\alpha)}
$$

which is invariant and homogeneous of degree $2 \gamma$. In the case that the reflection group $G$ is the group $\mathbb{Z}_{2}^{d}$ of sign changes, the weight function $\omega_{k}$ is a product function of the form $\prod_{j=1}^{d}\left|x_{j}\right|^{k_{j}}, k_{j} \geq 0$. We denote by $c_{k}$ the Mehta-type constant defined by

$$
c_{k}=\int_{\mathbb{R}^{d}} e^{-\|x\|^{2} / 2} \omega_{k}(x) d x .
$$

We note that Etingof (cf. [18]) has given a derivation of the Mehta-type constant valid for all finite reflection groups.

In the following, we denote by

$C\left(\mathbb{R}^{d}\right)$ : the space of continuous functions on $\mathbb{R}^{d}$.

$C^{p}\left(\mathbb{R}^{d}\right)$ : the space of functions of class $C^{p}$ on $\mathbb{R}^{d}$.

$C_{b}^{p}\left(\mathbb{R}^{d}\right)$ : the space of bounded functions of class $C^{p}$.

$\mathscr{E}\left(\mathbb{R}^{d}\right)$ : the space of $C^{\infty}$ functions on $\mathbb{R}^{d}$.

$\mathcal{S}\left(\mathbb{R}^{d}\right)$ : the Schwartz space of rapidly decreasing functions on $\mathbb{R}^{d}$.

$D\left(\mathbb{R}^{d}\right)$ : the space of $C^{\infty}$ functions on $\mathbb{R}^{d}$ which are of compact support.

$\mathcal{S}^{\prime}\left(\mathbb{R}^{d}\right)$ : the space of temperate distributions on $\mathbb{R}^{d}$.

2.2. The Dunkl Operators. Let $k: R \rightarrow \mathbb{C}$ be a multiplicity function on $R$ and $R_{+}$a fixed positive root system of $R$. Then, the Dunkl operators $T_{j}, 1 \leq j \leq d$, are defined on $C^{1}\left(\mathbb{R}^{d}\right)$ by

$$
T_{j} f(x)=\frac{\partial}{\partial x_{j}} f(x)+\sum_{\alpha \in R_{+}} k(\alpha) \alpha_{j} \frac{f(x)-f\left(\sigma_{\alpha}(x)\right)}{\langle\alpha, x\rangle},
$$

where $\alpha=\left(\alpha_{1}, \alpha_{2}, \ldots, \alpha_{d}\right)$. Similarly, as ordinary derivatives, each $T_{j}$ satisfies for all $f, g$ in $C^{1}\left(\mathbb{R}^{d}\right)$ and at least one of them is $G$-invariant,

$$
T_{j}(f g)=\left(T_{j} f\right) g+f\left(T_{j} g\right),
$$

and for all $f$ in $C_{b}^{1}\left(\mathbb{R}^{d}\right)$ and $g$ in $\delta\left(\mathbb{R}^{d}\right)$,

$$
\int_{\mathbb{R}^{d}} T_{j} f(x) g(x) \omega_{k}(x) d x=-\int_{\mathbb{R}^{d}} f(x) T_{j} g(x) \omega_{k}(x) d x .
$$

Furthermore, according to $[1,14]$, the Dunkl operators $T_{j}, 1 \leq j \leq d$ commute, and there exists the so-called Dunkl's intertwining operator $V_{k}$ such that $T_{j} V_{k}=V_{k}\left(\partial / \partial x_{j}\right)$ for $1 \leq j \leq d$ and $V_{k}(1)=1$. We define the Dunkl-Laplace operator $\triangle_{k}$ on $\mathbb{R}^{d}$ by

$$
\begin{aligned}
\triangle_{k} f(x):= & \sum_{j=1}^{d} T_{j}^{2} f(x) \\
=\Delta f(x)+2 \sum_{\alpha \in R^{+}} k(\alpha)\left(\frac{\langle\nabla f(x), \alpha\rangle}{\langle\alpha, x\rangle}\right. & \left.-\frac{f(x)-f\left(\sigma_{\alpha}(x)\right)}{\langle\alpha, x\rangle^{2}}\right),
\end{aligned}
$$

where $\triangle$ and $\nabla$ are the usual Euclidean Laplacian and nabla operators on $\mathbb{R}^{d}$, respectively. Since the Dunkl operators commute, their joint eigenvalue problem is significant, and for each $y \in \mathbb{R}^{d}$, the system

$$
T_{j} u(x, y)=y_{j} u(x, y), \quad j=1, \ldots, d, u(0, y)=1
$$

admits a unique analytic solution $K(x, y), x \in \mathbb{R}^{d}$, called the Dunkl kernel, which has a holomorphic extension to $\mathbb{C}^{d} \times \mathbb{C}^{d}$. For $x, y \in \mathbb{C}^{d}$, the kernel satisfies

(a) $K(x, y)=K(y, x)$,

(b) $K(\lambda x, y)=K(x, \lambda y)$ for $\lambda \in \mathbb{C}$,

(c) $K(g x, g y)=K(x, y)$ for $g \in G$.

2.3. The Dunkl Transform. For functions $f$ on $\mathbb{R}^{d}$, we define $L^{p}$-norms of $f$ with respect to $\omega_{k}(x) d x$ as

$$
\|f\|_{L_{k}^{p}\left(\mathbb{R}^{d}\right)}=\left(\int_{\mathbb{R}^{d}}|f(x)|^{p} \omega_{k}(x) d x\right)^{1 / p},
$$

if $1 \leq p<\infty$ and $\|f\|_{L_{k}^{\infty}\left(\mathbb{R}^{d}\right)}=\operatorname{ess~sup}_{x \in \mathbb{R}^{d}}|f(x)|$. We denote by $L_{k}^{p}\left(\mathbb{R}^{d}\right)$ the space of all measurable functions $f$ on $\mathbb{R}^{d}$ with finite $L_{k}^{p}$-norm.

The Dunkl transform $\mathscr{F}_{D}$ on $L_{k}^{1}\left(\mathbb{R}^{d}\right)$ is given by

$$
\mathscr{F}_{D}(f)(y)=\frac{1}{c_{k}} \int_{\mathbb{R}^{d}} f(x) K(x,-i y) \omega_{k}(x) d x .
$$


Some basic properties are the following (cf. $[5,6])$. For all $f \in L_{k}^{1}\left(\mathbb{R}^{d}\right)$,

(a) $\left\|\mathscr{F}_{D}(f)\right\|_{L_{k}^{\infty}\left(\mathbb{R}^{d}\right)} \leq c_{k}^{-1}\|f\|_{L_{k}^{1}\left(\mathbb{R}^{d}\right)}$,

(b) $\mathscr{F}_{D}(f(\cdot / \lambda))(y)=\lambda^{2 \gamma+d} \mathscr{F}_{D}(f)(\lambda y)$ for $\lambda>0$,

(c) if $\mathscr{F}_{D}(f)$ belongs to $L_{k}^{1}\left(\mathbb{R}^{d}\right)$, then

$f(y)=\frac{1}{c_{k}} \int_{\mathbb{R}^{d}} \mathscr{F}_{D}(f)(x) K(i x, y) \omega_{k}(x) d x$, a.e.,

and moreover, for all $f \in \mathcal{S}\left(\mathbb{R}^{d}\right)$,

(d) $\mathscr{F}_{D}\left(T_{j} f\right)(y)=i y_{j} \mathscr{F}_{D}(f)(y)$,

(e) if we define $\overline{\mathscr{F}_{D}}(f)(y)=\mathscr{F}_{D}(f)(-y)$, then

$$
\mathscr{F}_{D} \overline{\mathscr{F}_{D}}=\overline{\mathscr{F}_{D}} \mathscr{F}_{D}=I \text { d. }
$$

Proposition 1. The Dunkl transform $\mathscr{F}_{D}$ is a topological isomorphism from $\mathcal{S}\left(\mathbb{R}^{d}\right)$ onto itself, and for all $f$ in $\mathcal{S}\left(\mathbb{R}^{d}\right)$,

$$
\int_{\mathbb{R}^{d}}|f(x)|^{2} \omega_{k}(x) d x=\int_{\mathbb{R}^{d}}\left|\mathscr{F}_{D}(f)(\xi)\right|^{2} \omega_{k}(\xi) d \xi .
$$

In particular, the Dunkl transform $f \rightarrow \mathscr{F}_{D}(f)$ can be uniquely extended to an isometric isomorphism on $L_{k}^{2}\left(\mathbb{R}^{d}\right)$.

We define the tempered distribution $\mathscr{T}_{f}$ associated with $f \in L_{k}^{p}\left(\mathbb{R}^{d}\right)$ by

$$
\left\langle\mathscr{T}_{f}, \phi\right\rangle=\int_{\mathbb{R}^{d}} f(x) \phi(x) \omega_{k}(x) d x,
$$

for $\phi \in \mathcal{S}\left(\mathbb{R}^{d}\right)$ and denote by $\langle f, \phi\rangle_{k}$ the integral in the right hand side.

Definition 2. The Dunkl transform $\mathscr{F}_{D}(\tau)$ of a distribution $\tau \in \mathcal{S}^{\prime}\left(\mathbb{R}^{d}\right)$ is defined by

$$
\left\langle\mathscr{F}_{D}(\tau), \phi\right\rangle=\left\langle\tau, \mathscr{F}_{D}(\phi)\right\rangle,
$$

for $\phi \in \mathcal{S}\left(\mathbb{R}^{d}\right)$.

In particular, for $f \in L_{k}^{p}\left(\mathbb{R}^{d}\right)$, it follows that for $\phi \in \mathcal{S}\left(\mathbb{R}^{d}\right)$,

$$
\begin{aligned}
\left\langle\mathscr{F}_{D}(f), \phi\right\rangle & =\left\langle\mathscr{F}_{D}\left(\mathscr{T}_{f}\right), \phi\right\rangle=\left\langle\mathscr{T}_{f}, \mathscr{F}_{D}(\phi)\right\rangle \\
& =\left\langle f, \mathscr{F}_{D}(\phi)\right\rangle_{k} .
\end{aligned}
$$

Proposition 3. The Dunkl transform $\mathscr{F}_{D}$ is a topological isomorphism from $\delta^{\prime}\left(\mathbb{R}^{d}\right)$ onto itself.

2.4. The Dunkl Convolution. By using the Dunkl kernel in Section 2.2, we introduce a generalized translation and a convolution structure in our Dunkl setting. For a function $f \in \mathcal{S}\left(\mathbb{R}^{d}\right)$ and $y \in \mathbb{R}^{d}$, the Dunkl translation $\tau_{y} f$ is defined by

$$
\tau_{y} f(x)=\frac{1}{c_{k}} \int_{\mathbb{R}^{d}} \mathscr{F}_{D}(f)(z) K(i x, z) K(i y, z) \omega_{k}(z) d z .
$$

Clearly, $\tau_{y} f(x)=\tau_{x} f(y)$, and by using the Dunkl's intertwining operator $V_{k}, \tau_{y} f$ is related to the usual translation as $\tau_{y} f(x)=\left(V_{k}\right)_{x}\left(V_{k}\right)_{y}\left(\left(V_{k}\right)^{-1}(f)(x+y)\right)$ (cf. [11, 17]). Hence, $\tau_{y}$ can also be defined for $f \in \mathscr{E}\left(\mathbb{R}^{d}\right)$. We define the Dunkl convolution product $f *_{D} g$ of functions $f, g \in \mathcal{S}\left(\mathbb{R}^{d}\right)$ as follows:

$$
f *_{D} g(x)=\int_{\mathbb{R}^{d}} \tau_{x} f(-y) g(y) \omega_{k}(y) d y .
$$

This convolution is commutative and associative (cf. [17]).

Since $\mathscr{F}_{D}\left(\tau_{y} f\right)(x)=K(i x, y) \mathscr{F}_{D}(f)(x)$ by the previous definition of $\tau_{y} f$, it follows that

(a) for all $f, g \in D\left(\mathbb{R}^{d}\right)$ (resp., $\left.\mathcal{S}\left(\mathbb{R}^{d}\right)\right), f *_{D} g$ belongs to $D\left(\mathbb{R}^{d}\right)\left(\right.$ resp. $\left.\mathcal{S}\left(\mathbb{R}^{d}\right)\right)$ and

$$
\mathscr{F}_{D}\left(f *_{D} g\right)(y)=\mathscr{F}_{D}(f)(y) \mathscr{F}_{D}(g)(y) .
$$

Moreover, as pointed in [16] and Sections 4 and 7, the operator $f \rightarrow f *_{D} g$ is bounded on $L_{k}^{p}\left(\mathbb{R}^{d}\right), 1 \leq p \leq \infty$, provided that $g$ is a radial function in $L_{k}^{1}\left(\mathbb{R}^{d}\right)$ or an arbitrary function in $L_{k}^{1}\left(\mathbb{R}^{d}\right)$ for $G=\mathbb{Z}_{2}^{d}$. Hence, the standard argument yields the following Young's inequality.

(b) Let $1 \leq p, q, r \leq \infty$ such that $1 / p+1 / q-1 / r=1$. Assume that $f \in L_{k}^{p}\left(\mathbb{R}^{d}\right)$ and $g \in L_{k}^{q}\left(\mathbb{R}^{d}\right)$. If $\left\|\tau_{x} g\right\|_{L_{k}^{q}\left(\mathbb{R}^{d}\right)} \leq C\|g\|_{L_{k}^{q}\left(\mathbb{R}^{d}\right)}$ for all $x \in \mathbb{R}^{d}$, then $f *_{D} g \in$ $L_{k}^{r}\left(\mathbb{R}^{d}\right)$ and

$$
\left\|f *_{D} g\right\|_{L_{k}^{r}\left(\mathbb{R}^{d}\right)} \leq C\|f\|_{L_{k}^{p}\left(\mathbb{R}^{d}\right)}\|g\|_{L_{k}^{q}\left(\mathbb{R}^{d}\right)} .
$$

Definition 4. The Dunkl convolution product of a distribution $S$ in $\mathcal{S}^{\prime}\left(\mathbb{R}^{d}\right)$ and a function $\phi$ in $\delta\left(\mathbb{R}^{d}\right)$ is the function $S *_{D} \phi$ defined by

$$
S *_{D} \phi(x)=\left\langle S_{y}, \tau_{-y} \phi(x)\right\rangle .
$$

Proposition 5. Let $f$ be in $L_{k}^{p}\left(\mathbb{R}^{d}\right), 1 \leq p \leq \infty$, and $\phi$ in $\mathcal{S}\left(\mathbb{R}^{d}\right)$. Then, the distribution $\mathscr{T}_{f}{ }_{D} \phi$ is given by the function $f *_{D} \phi$. If one assumes that $\phi$ is arbitrary for $d=1$ and radial for $d \geq 2$, then $\mathscr{T}_{f}{ }^{*} D \phi$ belongs to $L_{k}^{p}\left(\mathbb{R}^{d}\right)$. Moreover, for all $\psi \in \mathcal{S}\left(\mathbb{R}^{d}\right)$,

$$
\left\langle\mathscr{T}_{f}{ }_{D} \phi, \psi\right\rangle=\left\langle\check{f}, \phi *_{D} \check{\psi}\right\rangle_{k},
$$

where $\check{\psi}(x)=\psi(-x)$, and

$$
\mathscr{F}_{D}\left(\mathscr{T}_{f} *_{D} \phi\right)=\mathscr{F}_{D}\left(\mathscr{T}_{f}\right) \mathscr{F}_{D}(\phi) .
$$

For each $u \in \mathcal{S}^{\prime}\left(\mathbb{R}^{d}\right)$, we define the distributions $T_{j} u, 1 \leq j \leq d$, by

$$
\left\langle T_{j} u, \psi\right\rangle=-\left\langle u, T_{j} \psi\right\rangle,
$$


for all $\psi \in \mathcal{S}\left(\mathbb{R}^{d}\right)$. Then, $\left\langle\triangle_{k} u, \psi\right\rangle=\left\langle u, \triangle_{k} \psi\right\rangle$, and these distributions satisfy the following properties (see Section 2.3 (d)):

$$
\begin{gathered}
\mathscr{F}_{D}\left(T_{j} u\right)=i y_{j} \mathscr{F}_{D}(u), \\
\mathscr{F}_{D}\left(\triangle_{k} u\right)=-\|y\|^{2} \mathscr{F}_{D}(u) .
\end{gathered}
$$

In the following, we denote $\mathscr{T}_{f}$ given by (15) by $f$ for simplicity.

\section{3. $\dot{\mathscr{B}}_{p, q}^{s, k}, \dot{\mathscr{F}}_{p, q}^{s, k}\left(\mathbb{R}^{d}\right)$, and $\dot{\mathscr{H}}_{p, k}^{s}$ Spaces and \\ Basic Properties}

One of the main tools in this paper is the homogeneous Littlewood-Paley decompositions of distributions associated with the Dunkl operators into dyadic blocs of frequencies.

Lemma 6. Let one define by $\mathscr{C}$ the ring of center 0 , of small radius $1 / 2$, and great radius 2 . There exist two radial functions $\psi$ and $\varphi$ the values of which are in the interval $[0,1]$ belonging to $D\left(\mathbb{R}^{d}\right)$ such that

$$
\begin{gathered}
\operatorname{supp} \psi \subset B(0,1), \quad \operatorname{supp} \varphi \subset \mathscr{C} \\
\forall \xi \in \mathbb{R}^{d}, \quad \psi(\xi)+\sum_{j \geq 0} \varphi\left(2^{-j} \xi\right)=1 \\
\forall \xi \in \mathscr{C}, \quad \sum_{j \in \mathbb{Z}} \varphi\left(2^{-j} \xi\right)=1 \\
|n-m| \geq 2 \Longrightarrow \operatorname{supp} \varphi\left(2^{-n} \cdot\right) \cap \operatorname{supp} \varphi\left(2^{-m} \cdot\right)=\emptyset \\
j \geq 1 \Longrightarrow \operatorname{supp} \psi \cap \operatorname{supp} \varphi\left(2^{-j} \cdot\right)=\emptyset .
\end{gathered}
$$

Notations. We denote by

$$
\begin{array}{r}
\Delta_{j} f=\mathscr{F}_{D}^{-1}\left(\varphi\left(\frac{\xi}{2^{j}}\right) \mathscr{F}_{D}(f)\right), \quad S_{j} f=\sum_{n \leq j-1} \Delta_{n} f, \\
\forall j \in \mathbb{Z} .
\end{array}
$$

The distribution $\Delta_{j} f$ is called the $j$ th dyadic block of the homogeneous Littlewood-Paley decomposition of $f$ associated with the Dunkl operators.

Throughout this paper, we define $\phi$ and $\chi$ by $\phi=\mathscr{F}_{D}^{-1}(\varphi)$ and $\chi=\mathscr{F}_{D}^{-1}(\psi)$.

When dealing with the Littlewood-Paley decomposition, it is convenient to introduce the functions $\widetilde{\psi}$ and $\widetilde{\varphi}$ belonging to $D\left(\mathbb{R}^{d}\right)$ such that $\widetilde{\psi} \equiv 1$ on supp $\psi$ and $\widetilde{\varphi} \equiv 1$ on supp $\varphi$.

Remark 7. We remark that

$$
\begin{gathered}
\mathscr{F}_{D}\left(S_{j} f\right)(\xi)=\widetilde{\psi}\left(\frac{\xi}{2^{j}}\right) \mathscr{F}_{D}\left(S_{j} f\right)(\xi), \\
\mathscr{F}_{D}\left(\Delta_{j} f\right)(\xi)=\widetilde{\varphi}\left(\frac{\xi}{2^{j}}\right) \mathscr{F}_{D}\left(\Delta_{j} f\right)(\xi) .
\end{gathered}
$$

We put

$$
\widetilde{\phi}=\mathscr{F}_{D}^{-1}(\widetilde{\varphi}), \quad \tilde{\chi}=\mathscr{F}_{D}^{-1}(\widetilde{\psi}) .
$$

Definition 8. Let one denote by $\delta_{h, k}^{\prime}\left(\mathbb{R}^{d}\right)$ the space of tempered distribution such that

$$
\lim _{j \rightarrow-\infty} S_{j} u=0 \quad \text { in } \mathcal{S}^{\prime}\left(\mathbb{R}^{d}\right) .
$$

On the follow, we define analogues of the homogeneous Besov, Triebel-Lizorkin, and Riesz potential spaces associated with the Dunkl operators on $\mathbb{R}^{d}$ and obtain their basic properties.

From now, we make the convention that for all nonnegative sequence $\left\{a_{q}\right\}_{q \in \mathbb{Z}}$, the notation $\left(\sum_{q} a_{q}^{r}\right)^{1 / r}$ stands for $\sup _{q} a_{q}$ in the case $r=\infty$.

Definition 9. Let $s \in \mathbb{R}$ and $p, q \in[1, \infty]$. The homogeneous Dunkl-Besov spaces $\dot{\mathscr{B}}_{p, q}^{s, k}\left(\mathbb{R}^{d}\right)$ are the space of distribution in $\delta_{h, k}^{\prime}\left(\mathbb{R}^{d}\right)$ such that

$$
\|f\|_{\dot{S}_{p, q}^{s, k}\left(\mathbb{R}^{d}\right)}:=\left(\sum_{j \in \mathbb{Z}}\left(2^{s j}\left\|\Delta_{j} f\right\|_{L_{k}^{p}\left(\mathbb{R}^{d}\right)}\right)^{q}\right)^{1 / q}<\infty .
$$

Definition 10. Let $s \in \mathbb{R}$ and $1 \leq p, q \leq \infty$, the homogeneous Dunkl-Triebel-Lizorkin space $\underset{\mathscr{F}_{p, q}^{s, k}}{\dot{1}}\left(\mathbb{R}^{d}\right)$ is the space of distribution in $\mathcal{S}_{h, k}^{\prime}\left(\mathbb{R}^{d}\right)$ such that

$$
\|f\|_{\dot{\mathscr{F}}_{p, q}^{s, k}\left(\mathbb{R}^{d}\right)}:=\left\|\left(\sum_{j \in \mathbb{Z}} 2^{s j q}\left|\Delta_{j} f\right|^{q}\right)^{1 / q}\right\|_{L_{k}^{p}\left(\mathbb{R}^{d}\right)}<\infty .
$$

Let us recall that the operators $\left(-\triangle_{k}\right)^{s / 2}$ and $\left(I-\triangle_{k}\right)^{s / 2}$ have been defined, respectively, by (cf. [19])

$$
\begin{gathered}
\left(-\triangle_{k}\right)^{s / 2} f=\mathscr{F}_{D}^{-1}\left(\|\cdot\|^{s} \mathscr{F}_{D} f\right), \\
\left(I-\triangle_{k}\right)^{s / 2} f=\mathscr{F}_{D}^{-1}\left(\left(1+\|\cdot\|^{2}\right)^{s / 2} \mathscr{F}_{D} f\right) .
\end{gathered}
$$

The operators $\left(I-\triangle_{k}\right)^{-s / 2}$, for $s>0$, are called Dunkl-Bessel potential operators, and they are given by Dunkl convolution with the Dunkl-Bessel potential

$$
\left(I-\triangle_{k}\right)^{-s / 2} f=f *_{D} B_{k, s},
$$

where

$$
B_{k, s}(y)=\frac{1}{\Gamma(s / 2)} \int_{0}^{\infty} e^{-t} e^{-\|y\|^{2} / 4 t} t^{(s-d-2 \gamma) / 2} \frac{d t}{t} .
$$

We note that $B_{k, s}(y) \geq 0$ for all $y \in \mathbb{R}^{d}, B_{k, s} \in L_{k}^{1}\left(\mathbb{R}^{d}\right)$, and

$$
B_{k, s}(y) \leq C\|y\|^{s-d-2 \gamma} e^{-\|y\|^{2} / 2}, \quad\|y\|>0 .
$$

Definition 11. For $s \in \mathbb{R}$ and $1 \leq p \leq \infty$, the DunklBessel potential space $H_{p, k}^{s}\left(\mathbb{R}^{d}\right)$ is defined as the space $\left(I-\triangle_{k}\right)^{s / 2}\left(L_{k}^{p}\left(\mathbb{R}^{d}\right)\right)$, equipped with the norm $\|f\|_{H_{p, k}^{s}\left(\mathbb{R}^{d}\right)}=$ $\left\|\left(I-\triangle_{k}\right)^{s / 2} f\right\|_{L_{k}^{p}\left(\mathbb{R}^{d}\right)}$. 
Furthermore, $p=2, H_{2, k}^{s}\left(\mathbb{R}^{d}\right):=H_{k}^{s}\left(\mathbb{R}^{d}\right)$.

Definition 12. The operators $\left(-\triangle_{k}\right)_{k}^{-s / 2}, 0<s<d+2 \gamma$, are called Dunkl-Riesz potentials operators, and one has

$$
\left(-\triangle_{k}\right)^{-s / 2} f=R_{k, s}{ }_{D} f,
$$

where $R_{k, s}$ is the Dunkl-Riesz potential given by

$$
R_{k, s}(y):=C(k, s, d)\|y\|^{s-d-2 \gamma},
$$

$$
\text { where } C(k, s, d)=\frac{\Gamma((d+2 \gamma-s) / 2)}{2^{(d+2 \gamma-s) / 2} \Gamma(s / 2)} .
$$

Definition 13. For $s \in \mathbb{R}$ and $1 \leq p \leq \infty$, the homogeneous Dunkl-Riesz potential space $\dot{\mathscr{H}}_{p, k}^{s}\left(\mathbb{R}^{d}\right)$ is defined as the space $\left(-\triangle_{k}\right)^{s / 2}\left(L_{k}^{p}\left(\mathbb{R}^{d}\right)\right)$, equipped with the norm $\|f\|_{\dot{\mathscr{C}}_{p, k}^{s}\left(\mathbb{R}^{d}\right)}=$ $\left\|\left(-\triangle_{k}\right)^{s / 2} f\right\|_{L_{k}^{p}\left(\mathbb{R}^{d}\right)}$.

Proposition 14. Let $q \in(1, \infty)$, and let $s \in \mathbb{R}$ such that $0<s<(d+2 \gamma) / q$, then one has

$$
\begin{aligned}
\dot{\mathscr{B}}_{q, q}^{s, k} & \left(\mathbb{R}^{d}\right) \\
& =\dot{\mathscr{F}}_{q, q}^{s, k}\left(\mathbb{R}^{d}\right) \hookrightarrow \dot{\mathscr{F}}_{q, \infty}^{s, k}\left(\mathbb{R}^{d}\right) \hookrightarrow \dot{\mathscr{F}}_{\infty, \infty}^{s-(d+2 \gamma) / q, k}\left(\mathbb{R}^{d}\right), \\
\dot{\mathscr{H}}_{q, k}^{s} & \left(\mathbb{R}^{d}\right) \\
& =\dot{\mathscr{F}}_{q, 2}^{s, k}\left(\mathbb{R}^{d}\right) \hookrightarrow \dot{\mathscr{F}}_{q, \infty}^{s, k}\left(\mathbb{R}^{d}\right) \hookrightarrow \dot{\mathscr{F}}_{\infty, \infty}^{s-(d+2 \gamma) / q, k}\left(\mathbb{R}^{d}\right) .
\end{aligned}
$$

Proof. We obtain these results by similar ideas used in the nonhomogeneous case. (cf. [12]).

Theorem 15. Let $a, b>0$, and let $q_{1}, q_{2} \in[1, \infty]$. Let $\theta=a /(a+b) \in(0,1)$, and let $1 / p=(1-\theta) / q_{1}+$ $\theta / q_{2}$. Then, there exists a constant $C$ such that for every $f \in \dot{\mathscr{F}}_{q_{1}, \infty}^{a, k}\left(\mathbb{R}^{d}\right) \cap \dot{\mathscr{F}}_{q_{2}, \infty}^{-b, k}\left(\mathbb{R}^{d}\right)$, then one has

$$
|f(x)| \leq C\left(\sup _{j \in \mathbb{Z}} 2^{a j}\left|\Delta_{j} f(x)\right|\right)^{1-\theta}\left(\sup _{j \in \mathbb{Z}} 2^{-b j}\left|\Delta_{j} f(x)\right|\right)^{\theta} .
$$

In particular, one gets

$$
\|f\|_{L_{k}^{p}\left(\mathbb{R}^{d}\right)} \leq C\|f\|_{\dot{F}_{q_{1}, \infty}^{a, k}\left(\mathbb{R}^{d}\right)}^{1-\theta}\|f\|_{\dot{\mathscr{F}}_{q_{2}, \infty}^{-b, k}\left(\mathbb{R}^{d}\right)}^{\theta} .
$$

Proof. Let $f$ be a Schwartz class, we have

$$
\begin{aligned}
|f(x)| \leq & \sum_{j \in \mathbb{Z}}\left|\Delta_{j} f(x)\right| \\
\leq & \sum_{j \in \mathbb{Z}} \min \left(2^{-a j} \sup _{j \in \mathbb{Z}}\left(2^{a j}\left|\Delta_{j} f(x)\right|\right),\right. \\
& \left.2^{j b} \sup _{j \in \mathbb{Z}}\left(2^{-j b}\left|\Delta_{j} f(x)\right|\right)\right) .
\end{aligned}
$$

We define $N(x)$ as the largest index such that

$$
2^{j b} \sup _{j \in \mathbb{Z}}\left(2^{-j b}\left|\Delta_{j} f(x)\right|\right) \leq 2^{-a j} \sup _{j \in \mathbb{Z}}\left(2^{a j}\left|\Delta_{j} f(x)\right|\right),
$$

and we write

$$
\begin{aligned}
& |f(x)| \\
& \leq \sum_{j \leq N(x)} 2^{j b} \sup _{j \in \mathbb{Z}}\left(2^{-j b}\left|\Delta_{j} f(x)\right|\right) \\
& \quad+\sum_{j>N(x)} 2^{-a j} \sup _{j \in \mathbb{Z}}\left(2^{a j}\left|\Delta_{j} f(x)\right|\right) \\
& \quad \leq C\left(\sup _{j \in \mathbb{Z}} 2^{a j}\left|\Delta_{j} f(x)\right|\right)^{b /(a+b)}\left(\sup _{j \in \mathbb{Z}} 2^{-b j}\left|\Delta_{j} f(x)\right|\right)^{a /(a+b)} .
\end{aligned}
$$

Thus, (42) is proved. In order to obtain (43), it is enough to apply Hölder's inequality in the expression previous since we have $\theta=a /(a+b) \in(0,1)$ and $1 / p=(1-\theta) / q_{1}+\theta / q_{2}$.

Corollary 16. Let $q \in(1, \infty)$, and let $s \in \mathbb{R}$ such that $0<s<(d+2 \gamma) / q$, then one has

$$
\begin{aligned}
& \|f\|_{L_{k}^{p}\left(\mathbb{R}^{d}\right)} \leq C\|f\|_{\dot{\mathscr{B}}_{\infty, \infty}^{-((2 \gamma+d) / q-s), k}\left(\mathbb{R}^{d}\right)}^{1-q / p}\|f\|_{\mathscr{B}_{q, q}^{s, k}\left(\mathbb{R}^{d}\right)^{\prime}}^{q / p}, \\
& \|f\|_{L_{k}^{p}\left(\mathbb{R}^{d}\right)} \leq C\|f\|_{\dot{\mathscr{B}}_{\infty, \infty}^{-((2 p+d) / q-s) k, k, k}\left(\mathbb{R}^{d}\right)}^{1-q / p}\|f\|_{\dot{\mathscr{H}}_{q, k}^{s},\left(\mathbb{R}^{d}\right)}^{q / p},
\end{aligned}
$$

where $p=q(2 \gamma+d) /(2 \gamma+d-q s)$.

Proof. We take $a=s>0,-b=s-(d+2 \gamma) / q<0, q_{1}=q$, and $q_{2}=\infty$, and we deduce the inequality (47) from the relations (43) and (40). In the same way, we deduce (48) from the relations (43) and (41).

Theorem 17 (see [13]). (1) Let $s>0$ and $p, r \in[1, \infty]$. Then, $\dot{\mathscr{B}}_{p, r}^{s, k}\left(\mathbb{R}^{d}\right) \cap L_{k}^{\infty}\left(\mathbb{R}^{d}\right)$ is an algebra, and there exists a positive constant $C$ such that

$$
\begin{aligned}
& \|u v\|_{\dot{\mathscr{S}}_{p, r}^{s, k}\left(\mathbb{R}^{d}\right)} \\
& \quad \leq C\left[\|u\|_{L_{k}^{\infty}\left(\mathbb{R}^{d}\right)}\|v\|_{\dot{\mathscr{B}}_{P, r}^{s, k}\left(\mathbb{R}^{d}\right)}+\|v\|_{L_{k}^{\infty}\left(\mathbb{R}^{d}\right)}\|u\|_{\dot{\mathscr{B}}_{p, r}^{s, k}\left(\mathbb{R}^{d}\right)}\right] .
\end{aligned}
$$

(2) Moreover, for any $\left(s_{1}, s_{2}\right)$, any $p_{2}$ and any $r_{2}$ such that $s_{1}+s_{2}>(d+2 \gamma) / p_{1}$ and $s_{1}<(d+2 \gamma) / p_{1}$, one has

$$
\begin{aligned}
& \|u v\|_{\dot{\mathscr{S}}_{p_{2}, r_{2}}^{s, k}\left(\mathbb{R}^{d}\right)} \\
& \quad \leq C\left[\|u\|_{\dot{\mathscr{B}}_{p_{1}, \infty}^{s_{1}, k}\left(\mathbb{R}^{d}\right)}\|v\|_{\dot{\mathscr{S}}_{P_{2}, r_{2}}^{s_{2}, k}\left(\mathbb{R}^{d}\right)}+\|u\|_{\dot{\mathscr{S}}_{P_{2}, r_{2}}^{s_{2}, k}\left(\mathbb{R}^{d}\right)}\|v\|_{\dot{\mathscr{S}}_{p_{1}, \infty}^{s_{1}, k}\left(\mathbb{R}^{d}\right)}\right],
\end{aligned}
$$

where $s=s_{1}+s_{2}-(d+2 \gamma) / p_{1}$. 
(3) Moreover, for any $\left(s_{1}, s_{2}\right)$, any $p_{2}$ and any $\left(r_{1}, r_{2}\right)$ such that $s_{1}+s_{2}>(d+2 \gamma) / p_{1}, s_{1}<(d+2 \gamma) / p_{1}, 1 / r_{1}+1 / r_{2}=1$, one has

$$
\begin{aligned}
& \|u v\|_{\dot{\mathscr{B}}_{p, \infty}^{s, k}\left(\mathbb{R}^{d}\right)} \\
& \quad \leq C\left[\|u\|_{\dot{\mathscr{B}}_{p_{1}, r_{1}}^{s_{1}, k}\left(\mathbb{R}^{d}\right)}\|v\|_{\dot{\mathscr{B}}_{p_{2}, r_{2}}^{s_{2}, k}\left(\mathbb{R}^{d}\right)}+\|u\|_{\dot{\mathscr{B}}_{P_{2}, r_{2}}^{s_{2}, k}\left(\mathbb{R}^{d}\right)}\|v\|_{\dot{\mathscr{B}}_{p_{1}, r_{1}}^{s_{1}, k}\left(\mathbb{R}^{d}\right)}\right] .
\end{aligned}
$$

(4) Moreover, for any $\left(s_{1}, s_{2}\right)$, any $\left(p_{1}, p_{2}, p\right)$ and any $\left(r_{1}, r_{2}\right)$ such that $s_{j}<(d+2 \gamma) / p_{j}, s_{1}+s_{2}>(d+2 \gamma)\left(1 / p_{1}+\right.$ $\left.1 / p_{2}-1 / p\right)$ and $p \geq \max \left(p_{1}, p_{2}\right)$, one has

$$
\|u v\|_{\dot{\mathscr{B}}_{p, r}^{s_{1,2}, k}\left(\mathbb{R}^{d}\right)} \leq C\|u\|_{\dot{\mathscr{B}}_{p_{1}, r_{1}}^{s_{1}, k}\left(\mathbb{R}^{d}\right)}\|v\|_{\dot{\mathscr{B}}_{p_{2}, r_{2}}^{s_{2}, k}\left(\mathbb{R}^{d}\right)^{j}}
$$

with $s_{1,2}=s_{1}+s_{2}-(d+2 \gamma)\left(1 / p_{1}+1 / p_{2}-1 / p\right)$ and $r=\max \left(r_{1}, r_{2}\right)$.

\section{A Primer to Real Interpolation Theory and Generalized Lorentz Spaces}

From now, we denote by $l^{q}(\mathbb{Z})$ the set of sequence $\left(a_{j}\right)_{j \in \mathbb{Z}}$ such that

$$
\left(\sum_{j \in \mathbb{Z}}\left|a_{j}\right|^{q}\right)^{1 / q}<\infty
$$

stands for $\sup _{j}\left|a_{j}\right|$ in the case $q=\infty$.

The theory of interpolation spaces was introduced in the early sixties by J. Lions and J. Peetre for the real method and by Caldéron for the complex method (cf. [20]).

In this section, we present the real method. There are many equivalent ways to define the method; we will present the discrete $J$-method and the $K$-method which are the simplest ones.

We consider two Banach spaces $A_{0}$ and $A_{1}$ which are continuously imbedded into a common topological vector space $V$ and $t>0$.

The $J$-method and the $K$-method consist to consider the $J$-functional and the $K$-functional defined on $A_{0} \cap A_{1}$ by

$$
\begin{gathered}
J\left(t, a ; A_{0}, A_{1}\right)=\max \left(\|a\|_{A_{0}}, t\|a\|_{A_{1}}\right), \\
K\left(t, a ; A_{0}, A_{1}\right)=\min \left(\left\|a_{0}\right\|_{A_{0}}+t\left\|a_{1}\right\|_{A_{1}}: a=a_{0}+a_{1}\right) .
\end{gathered}
$$

Definition 18 ( $J$-method of interpolation). For $0<\theta<1$ and $1 \leq q \leq \infty$, the interpolation space $\left[A_{0}, A_{1}\right]_{\theta, q, J}$ is defined as follows: $a \in\left[A_{0}, A_{1}\right]_{\theta, q, J}$ if and only if $a$ can be written as a sum $a=\sum_{j \in \mathbb{Z}} a_{j}$, where the series converge in $A_{0}+A_{1}$, each $a_{j}$ belongs to $A_{0} \bigcap A_{1}$ and $\left(2^{-j \theta} J\left(2^{j}, a_{j} ; A_{0}, A_{1}\right)\right)_{j \in \mathbb{Z}} \in l^{q}(\mathbb{Z})$.

The norm of $\left[A_{0}, A_{1}\right]_{\theta, q, J}$ is defined by

$$
\begin{aligned}
& \|a\|_{\left[A_{0}, A_{1}\right]_{\theta, q, J}} \\
& =\inf _{a=\sum_{j \in \mathbb{Z}} a_{j}}\left(\sum_{j \in \mathbb{Z}} 2^{-j \theta q}\left\|a_{j}\right\|_{A_{0}}^{q}\right)^{1 / q}+\left(\sum_{j \in \mathbb{Z}} 2^{j(1-\theta) q}\left\|a_{j}\right\|_{A_{1}}^{q}\right)^{1 / q} .
\end{aligned}
$$

Definition 19 (K-method of interpolation). For $0<\theta<1$ and $1 \leq q \leq \infty$, the space $\left[A_{0}, A_{1}\right]_{\theta, q, K}$ is defined by $a \in\left[A_{0}, A_{1}\right]_{\theta, q, K}$ if and only if $a \in A_{0}+A_{1}$, and $\left(2^{-j \theta} K\left(2^{j}, a ; A_{0}, A_{1}\right)\right)_{j \in \mathbb{Z}} \in l^{q}(\mathbb{Z})$.

The norm of $\left[A_{0}, A_{1}\right]_{\theta, q, K}$ is defined as follows:

$$
\|a\|_{\left[A_{0}, A_{1}\right]_{\theta, q, K}}=\left(\sum_{j \in \mathbb{Z}} 2^{-j \theta q} K\left(2^{j}, a ; A_{0}, A_{1}\right)^{q}\right)^{1 / q} .
$$

Proposition 20 (Equivalence theorem). For $0<\theta<1$ and $1 \leq q \leq \infty$, one has $\left[A_{0}, A_{1}\right]_{\theta, q, K}=\left[A_{0}, A_{1}\right]_{\theta, q, J}$.

Remark 21. In the following, we will denote this space by $\left[A_{0}, A_{1}\right]_{\theta, q}$.

Lemma 22. For $a=\sum_{j \in \mathbb{Z}} a_{j}$ and $\varrho>0$, with $\varrho \neq 1$, one has

$$
\begin{aligned}
\|a\|_{\left[A_{0}, A_{1}\right]_{\theta, q}} \leq & C(q, \theta, \varrho)\left(\sum_{j \in \mathbb{Z}} \varrho^{-j \theta q}\left\|a_{j}\right\|_{A_{0}}^{q}\right)^{(1-\theta) / q} \\
& \times\left(\sum_{j \in \mathbb{Z}} \varrho^{j(1-\theta) q}\left\|a_{j}\right\|_{A_{1}}^{q}\right)^{\theta / q} .
\end{aligned}
$$

Proposition 23. (i) For $\theta_{0} \neq \theta_{1}$, one has

$$
\left[\left[A_{0}, A_{1}\right]_{\theta_{0}, q_{0}},\left[A_{0}, A_{1}\right]_{\theta_{1}, q_{1}}\right]_{\theta, q}=\left[A_{0}, A_{1}\right]_{(1-\theta) \theta_{0}+\theta \theta_{1}, q} .
$$

(ii) For $\theta_{0}=\theta_{1}$, (58) is still valid if $1 / q=(1-\theta) / q_{0}+\theta / q_{1}$.

Proposition 24 (Duality theorem for the real method). One considers the dual spaces $A_{0}^{\prime}, A_{1}^{\prime}$ and $\left[A_{0}, A_{1}\right]_{\theta, q}^{\prime}$ for $0<\theta<1$ and $1 \leq q<\infty$ of the spaces $A_{0}, A_{1}$ and $\left[A_{0}, A_{1}\right]_{\theta, q}$. If $A_{0} \cap A_{1}$ is dense in $A_{0}$ and in $A_{1}$, one has $\left[A_{0}, A_{1}\right]_{\theta, q}^{\prime}=$ $\left[A_{0}^{\prime}, A_{1}^{\prime}\right]_{\theta, q^{\prime}}$, where $q^{\prime}$ is the conjugate component of $q$.

For any measurable function $f$ on $\mathbb{R}^{d}$, we define its distribution and rearrangement functions

$$
\begin{aligned}
d_{f, k}(\lambda) & :=\int_{\left\{x \in \mathbb{R}^{d}:|f(x)| \geq \lambda\right\}} \omega_{k}(x) d x, \\
f_{k}^{*}(s) & :=\inf \left\{\lambda: d_{f, k}(\lambda) \leq s\right\} .
\end{aligned}
$$

For $1 \leq p \leq \infty$ and $1 \leq q \leq \infty$, define

$$
\begin{aligned}
& \|f\|_{L_{k}^{p, q}\left(\mathbb{R}^{d}\right)} \\
& \quad= \begin{cases}\left(\int_{0}^{\infty}\left(s^{1 / p} f_{k}^{*}(s)\right)^{q} \frac{d s}{s}\right)^{1 / q} & \text { if } 1 \leq p, q<\infty \\
\sup _{s>0} s^{1 / p} f_{k}^{*}(s) & \text { if } 1 \leq p \leq \infty, q=\infty .\end{cases}
\end{aligned}
$$

The generalized Lorentz spaces $L_{k}^{p, q}\left(\mathbb{R}^{d}\right)$ is defined as the set of all measurable functions $f$ such that $\|f\|_{L_{k}^{p, q}\left(\mathbb{R}^{d}\right)}<\infty$. 
Proposition 25. (i) For $1<p<\infty, 1 \leq q \leq \infty$

$$
L_{k}^{p, q}\left(\mathbb{R}^{d}\right)=\left[L_{k}^{1}\left(\mathbb{R}^{d}\right), L_{k}^{\infty}\left(\mathbb{R}^{d}\right)\right]_{\theta, q},
$$

with $1 / p=1-\theta$.

(ii) For $p_{0} \neq p_{1}$, one has

$$
\begin{aligned}
& {\left[L_{k}^{p_{0}, q_{0}}\left(\mathbb{R}^{d}\right), L_{k}^{p_{1}, q_{1}}\left(\mathbb{R}^{d}\right)\right]_{\theta, q}=\left[L_{k}^{p_{0}}\left(\mathbb{R}^{d}\right), L_{k}^{p_{1}}\left(\mathbb{R}^{d}\right)\right]_{\theta, q}} \\
& \quad=L_{k}^{p, q}\left(\mathbb{R}^{d}\right),
\end{aligned}
$$

with $1 / p=(1-\theta) / p_{0}+\theta / p_{1}$.

(iii) In the case $p_{0}=p_{1}=p$, one has

$$
\left[L_{k}^{p, q_{0}}\left(\mathbb{R}^{d}\right), L_{k}^{p, q_{1}}\left(\mathbb{R}^{d}\right)\right]_{\theta, q}=L_{k}^{p, q}\left(\mathbb{R}^{d}\right),
$$

with $1 / q=(1-\theta) / q_{0}+\theta / q_{1}$.

(iv) If $1 \leq p \leq \infty$ and $1 \leq q_{1}<q_{2} \leq \infty$, then

$$
L_{k}^{p, q_{1}}\left(\mathbb{R}^{d}\right) \hookrightarrow L_{k}^{p, q_{2}}\left(\mathbb{R}^{d}\right) .
$$

Proof. We obtain these results by similar ideas used in the Euclidean case.

Proposition 26. (i) Let $1<p<\infty, 1 \leq q \leq \infty$. Then, there exists a constant $C$ such that every $f \in L_{k}^{p, q}\left(\mathbb{R}^{d}\right)$ can be decomposed as $f=\sum_{j \in \mathbb{Z}} f_{j}$, where

$$
\begin{aligned}
& \left\|\left(2^{-j(p-1) / p}\left\|f_{j}\right\|_{L_{k}^{1}\left(\mathbb{R}^{d}\right)}\right)\right\|_{l^{r}}+\left\|\left(2^{j / p}\left\|f_{j}\right\|_{L_{k}^{\infty}\left(\mathbb{R}^{d}\right)}\right)\right\|_{l^{r}} \\
& \leq C\|f\|_{L_{k}^{p, q}\left(\mathbb{R}^{d}\right)},
\end{aligned}
$$

the $f_{j}$ have disjoint supports if $j \neq n, f_{j} f_{n}=0$.

(ii) Let $1<p<\infty, 1 \leq q \leq \infty$. Then, there exists a constant $C$ such that every $f \in L_{k}^{p, q}\left(\mathbb{R}^{d}\right)$ and every $g \in$ $L_{k}^{p /(p-1), q /(q-1)}\left(\mathbb{R}^{d}\right)$, one has $f g \in L_{k}^{1}\left(\mathbb{R}^{d}\right)$ and

$$
\left|\int_{\mathbb{R}^{d}} f(x) g(x) \omega_{k}(x) d x\right| \leq C\|f\|_{L_{k}^{p, q}\left(\mathbb{R}^{d}\right)}\|g\|_{L_{k}^{p /(p-1), q /(q-1)}\left(\mathbb{R}^{d}\right)} .
$$

Proof. We obtain these results by similar ideas used in the Euclidean case.

\section{Inequalities for the Fractional Dunkl-Laplace Operators}

Lemma 27. Let $s$ be a real number such that $0<s<d+2 \gamma$, and let $1<p<q<\infty$ satisfy

$$
\frac{1}{p}-\frac{1}{q}=\frac{s}{2 \gamma+d}
$$

For $f \in L_{k}^{p}\left(\mathbb{R}^{d}\right)$, one has

$$
\left\|\left(I-\triangle_{k}\right)^{-s / 2} f\right\|_{L_{k}^{q}\left(\mathbb{R}^{d}\right)} \leq C\|f\|_{L_{k}^{p}\left(\mathbb{R}^{d}\right)} .
$$

Proof. We obtain this result by similar ideas used for the Dunkl-Riesz potential (cf. [21]).

Proposition 28. Let $s<(d+2 \gamma) / 2$ and $q=(2 d+4 \gamma) /(d+$ $2 \gamma-2 s)$. Then,

$$
\|f\|_{L_{k}^{q}\left(\mathbb{R}^{d}\right)} \leq C\left\|\left(I-\triangle_{k}\right)^{s / 2} f\right\|_{L_{k}^{2}\left(\mathbb{R}^{d}\right)}, \quad f \in H_{k}^{s}\left(\mathbb{R}^{d}\right) .
$$

Proof. Let us first observe that since $D\left(\mathbb{R}^{d}\right)$ is dense in $H_{k}^{s}\left(\mathbb{R}^{d}\right)$, it is enough to prove (69) for $f \in D\left(\mathbb{R}^{d}\right)$. Let $f, g \in D\left(\mathbb{R}^{d}\right)$. Then, we have

$$
\begin{aligned}
& \langle f, g\rangle_{L_{k}^{2}\left(\mathbb{R}^{d}\right)} \\
& =\left\langle\mathscr{F}_{D}(f), \mathscr{F}_{D}(g)\right\rangle_{L_{k}^{2}\left(\mathbb{R}^{d}\right)} \\
& =\int_{\mathbb{R}^{d}}\left(1+\|\xi\|^{2}\right)^{s / 2} \mathscr{F}_{D}(f)(\xi) \overline{\mathscr{F}_{D}(g)(\xi)}\left(1+\|\xi\|^{2}\right)^{-s / 2} \\
& \quad \times \omega_{k}(\xi) d \xi \\
& =\left\langle\left(I-\triangle_{k}\right)^{s / 2} f,\left(I-\triangle_{k}\right)^{-s / 2} g\right\rangle_{L_{k}^{2}\left(\mathbb{R}^{d}\right)}
\end{aligned}
$$

Hence,

$$
\left|\langle f, g\rangle_{L_{k}^{2}\left(\mathbb{R}^{d}\right)}\right| \leq\left\|\left(I-\triangle_{k}\right)^{s / 2} f\right\|_{L_{k}^{2}\left(\mathbb{R}^{d}\right)}\left\|\left(I-\triangle_{k}\right)^{-s / 2} g\right\|_{L_{k}^{2}\left(\mathbb{R}^{d}\right)} .
$$

Now, by the previous lemma we obtain

$$
\left|\langle f, g\rangle_{L_{k}^{2}\left(\mathbb{R}^{d}\right)}\right| \leq C\left\|\left(I-\triangle_{k}\right)^{s / 2} f\right\|_{L_{k}^{2}\left(\mathbb{R}^{d}\right)}\|g\|_{L_{k}^{p}\left(\mathbb{R}^{d}\right)},
$$

where $p=(2 d+4 \gamma) /(d+2 \gamma+2 s)$. Now, let us take $g=f^{q-1}$, with $1 / p+1 / q=1$, that is, $q=(2 d+4 \gamma) /(d+2 \gamma-2 s)$. Then, the relation (72) gives that

$$
\|f\|_{L_{k}^{q}\left(\mathbb{R}^{d}\right)}^{q} \leq C\left\|\left(I-\triangle_{k}\right)^{s / 2} f\right\|_{L_{k}^{2}\left(\mathbb{R}^{d}\right)}\|f\|_{L_{k}^{q}\left(\mathbb{R}^{d}\right)}^{q-1} .
$$

Thus, we obtain (69).

Proposition 29. Let $1 \leq p, p_{2}<\infty, 0<\theta<p<\infty$, $0<s<d+2 \gamma$, and $1<p_{1}<(d+2 \gamma) / s$. Then, one has the inequality

$$
\|f\|_{L_{k}^{p}\left(\mathbb{R}^{d}\right)} \leq C\left\|\left(I-\triangle_{k}\right)^{s / 2} f\right\|_{L_{k}^{p_{1}}\left(\mathbb{R}^{d}\right)}^{\theta / p}\|f\|_{L_{k}^{p_{2}}\left(\mathbb{R}^{d}\right)}^{(p-\theta) / p},
$$

with

$$
\theta\left(\frac{1}{p_{1}}-\frac{s}{d+2 \gamma}\right)+\frac{p-\theta}{p_{2}}=1
$$

Proof. Hölder's inequality yields

$$
\|f\|_{L_{k}^{p}\left(\mathbb{R}^{d}\right)}^{p} \leq C\|f\|_{L_{k}^{p_{0}}\left(\mathbb{R}^{d}\right)}^{\theta}\|f\|_{L_{k}^{p_{2}}\left(\mathbb{R}^{d}\right)}^{p-\theta},
$$


where

$$
\frac{1}{p_{0}}=\frac{1}{\theta}\left(1-\frac{1}{p}\right), \quad \frac{1}{p_{2}}=\frac{1}{(p-\theta) p} .
$$

Applying Lemma 27, with $p_{1}=\left((d+2 \gamma) p_{0}\right) /\left(d+2 \gamma+s p_{0}\right)$, we obtain the result.

Theorem 30. Let $1<q<\infty, 0<s<d+2 \gamma$ and $1<p_{1}<(d+2 \gamma) / s$. Then, the inequality

$$
\begin{aligned}
& \exp \left(\left(\frac{1}{q}+\frac{s}{d+2 \gamma}-\frac{1}{p_{1}}\right)\right. \\
& \left.\quad \times \int_{\mathbb{R}^{d}} \frac{|f(x)|^{q}}{\|f\|_{L_{k}^{q}\left(\mathbb{R}^{d}\right)}^{q}} \ln \left(\frac{|f(x)|^{q}}{\|f\|_{L_{k}^{q}\left(\mathbb{R}^{d}\right)}^{q}}\right) \omega_{k}(x) d x\right) \\
& \leq C \frac{\left\|\left(I-\triangle_{k}\right)^{s / 2} f\right\|_{L_{k}^{p_{1}}\left(\mathbb{R}^{d}\right)}}{\|f\|_{L_{k}^{q}\left(\mathbb{R}^{d}\right)}}
\end{aligned}
$$

holds for

$$
\frac{1}{q}+\frac{s}{d+2 \gamma}-\frac{1}{p_{1}}>0
$$

Proof. Using the convexity of the function $g(h)=$ $h \ln \left(\int_{\mathbb{R}^{d}}|f(x)|^{1 / h} \omega_{k}(x) d x\right)$, and the logarithmic Hölder's inequality proved by Merker [22], we obtain

$$
\begin{gathered}
\int_{\mathbb{R}^{d}} \frac{|f(x)|^{q}}{\|f\|_{L_{k}^{q}\left(\mathbb{R}^{d}\right)}^{q}} \ln \left(\frac{|f(x)|^{q}}{\|f\|_{L_{k}^{q}\left(\mathbb{R}^{d}\right)}^{q}}\right) \omega_{k}(x) d x \\
\leq \frac{p}{p-q} \ln \left(\frac{\|f\|_{L_{k}^{q}\left(\mathbb{R}^{d}\right)}^{q}}{\|f\|_{L_{k}^{q}\left(\mathbb{R}^{d}\right)}^{q}}\right),
\end{gathered}
$$

for $0<q<p \leq \infty$. We can choose $p=((d+2 \gamma) q) /(d+$ $2 \gamma-q s) \in(q, \infty)$ for $p_{2}=q$ and $\theta$ satisfying the condition of Proposition 29, and we get

$$
\begin{aligned}
& \int_{\mathbb{R}^{d}} \frac{|f(x)|^{q}}{\|f\|_{L_{k}^{q}\left(\mathbb{R}^{d}\right)}^{q}} \ln \left(\frac{|f(x)|^{q}}{\|f\|_{L_{k}^{q}\left(\mathbb{R}^{d}\right)}^{q}}\right) \omega_{k}(x) d x \\
& \quad \leq \frac{p}{p-q} \ln \left(\frac{\left(C\left\|\left(I-\triangle_{k}\right)^{s / 2} f\right\|_{L_{k}^{p_{1}}\left(\mathbb{R}^{d}\right)}^{\theta / p}\|f\|_{L_{k}^{p^{2}}\left(\mathbb{R}^{d}\right)}^{(p-\theta) / p}\right)^{q}}{\|f\|_{L_{k}^{q}\left(\mathbb{R}^{d}\right)}^{q}}\right) \\
& \quad \leq \frac{q \theta}{p-q} \ln \left(\frac{C\left\|\left(I-\triangle_{k}\right)^{s / 2} f\right\|_{L_{k}^{p_{1}\left(\mathbb{R}^{d}\right)}}}{\|f\|_{L_{k}^{q}\left(\mathbb{R}^{d}\right)}^{q}}\right) .
\end{aligned}
$$

By a simple calculation, we obtain the result.
Corollary 31. Let $0<s<d+2 \gamma$ and $1<q<(d+2 \gamma) / s$, $f \in H_{q, k}^{s}\left(\mathbb{R}^{d}\right)$ such that $\|f\|_{L_{k}^{q}\left(\mathbb{R}^{d}\right)}=1$, one has

$$
\begin{aligned}
& \exp \left(\frac{s}{d+2 \gamma} \int_{\mathbb{R}^{d}}|f(x)|^{q} \ln \left(|f(x)|^{q}\right) \omega_{k}(x) d x\right) \\
& \quad \leq C\left\|\left(I-\triangle_{k}\right)^{s / 2} f\right\|_{L_{k}^{q}\left(\mathbb{R}^{d}\right)^{\prime}} .
\end{aligned}
$$

Proof. It suffices to apply the previous theorem for $q=p_{1}$.

Lemma 32 (see [23]). One assumes that $G=\mathbb{Z}_{2}^{d}$. If $f \in L_{k}^{p_{1}, q_{1}}\left(\mathbb{R}^{d}\right), g \in L_{k}^{p_{2}, q_{2}}\left(\mathbb{R}^{d}\right)$, and $1 / p_{1}+1 / p_{2}>1$, then $f *_{D} g \in L_{k}^{p_{3}, q_{3}}\left(\mathbb{R}^{d}\right)$ where $1 / p_{3}=1 / p_{1}+1 / p_{2}-1$ and $q_{3} \geq 1$ is any number such that $1 / q_{3} \leq 1 / q_{1}+1 / q_{2}$. Moreover,

$$
\left\|f *{ }_{D} g\right\|_{L_{k}^{p_{3}, q_{3}}\left(\mathbb{R}^{d}\right)} \leq C\|f\|_{L_{k}^{p_{1}, q_{1}}\left(\mathbb{R}^{d}\right)}\|g\|_{L_{k}^{p_{2}, q_{2}}\left(\mathbb{R}^{d}\right)} .
$$

Remark 33. The analogues of this lemma for the general reflection group $G$, together with other additional results, will appear in a forthcoming paper.

Theorem 34. One assumes that $G=\mathbb{Z}_{2}^{d}$. Let $1 \leq p<\infty$, $1 \leq p_{2}, q, q_{1}, q_{2}<\infty, 0<\theta<q, 0<s<d+2 \gamma$, and $1<p_{1}<(d+2 \gamma) / s$. Then, the inequality

$$
\|f\|_{L_{k}^{p, q}\left(\mathbb{R}^{d}\right)} \leq C\left\|\left(I-\triangle_{k}\right)^{s / 2} f\right\|_{L_{k}^{p_{1}, q_{1}}\left(\mathbb{R}^{d}\right)}^{\theta / q}\|f\|_{L_{k}^{p_{2}, q_{2}}\left(\mathbb{R}^{d}\right)}^{(q-\theta) / q}
$$

holds for

$$
\begin{gathered}
\frac{\theta}{q_{1}}+\frac{q-\theta}{q_{2}}=1, \\
\theta\left(\frac{1}{p_{1}}-\frac{s}{d+2 \gamma}\right)+\frac{q-\theta}{p_{2}}=\frac{q}{p} .
\end{gathered}
$$

Proof. Applying the Hölder inequality and simple computation yields

$$
\|f\|_{L_{k}^{p, q}\left(\mathbb{R}^{d}\right)}^{q} \leq C\|f\|_{L_{k}^{p_{3}, q_{1}}\left(\mathbb{R}^{d}\right)}^{\theta}\|f\|_{L_{k}^{p_{2}, q_{2}}\left(\mathbb{R}^{d}\right)^{\prime}}^{q-\theta},
$$

where

$$
\begin{aligned}
& \frac{1}{p_{3}}=\frac{1}{p}-\frac{1}{q}+\frac{1}{q_{1}}, \\
& \frac{1}{p_{2}}=\frac{1}{p}-\frac{1}{q}+\frac{1}{q_{2}} .
\end{aligned}
$$

Note that

$$
f(x)=\left(I-\triangle_{k}\right)^{s / 2} f *_{D} B_{k, s}(x),
$$

where $B_{k, s}$ is the Dunkl-Bessel kernel defined by relation (36). From the relation (37), we see that $B_{k, s} \in$ $L_{k}^{(d+2 \gamma) /(d+2 \gamma-s), \infty}\left(\mathbb{R}^{d}\right)$. Using now Lemma 32, we deduce that

$$
\|f\|_{L_{k}^{p_{3}, q_{1}}\left(\mathbb{R}^{d}\right)} \leq C\left\|\left(I-\triangle_{k}\right)^{s / 2} f\right\|_{L_{k}^{p_{1}, q_{1}}\left(\mathbb{R}^{d}\right)},
$$


for

$$
\frac{1}{p_{3}}=\frac{1}{p_{1}}-\frac{s}{d+2 \gamma}, \quad 0<s<\frac{d+2 \gamma}{p_{1}} .
$$

The result then follows.

Now, we state the results for the Dunkl-Riesz potential operators. The proofs are essentially as for the Dunkl-Bessel potential operators. We will not repeat them.

Proposition 35. Let $s<(d+2 \gamma) / 2$ and $q=(2 d+4 \gamma) /(d+$ $2 \gamma-2 s)$. Then,

$$
\|f\|_{L_{k}^{q}\left(\mathbb{R}^{d}\right)} \leq C\left\|\left(-\triangle_{k}\right)^{s / 2} f\right\|_{L_{k}^{2}\left(\mathbb{R}^{d}\right)}, \quad f \in H_{k}^{s}\left(\mathbb{R}^{d}\right) .
$$

Proposition 36. Let $1 \leq p, p_{2}<\infty, 0<\theta<p<\infty$, $0<s<d+2 \gamma$, and $1<p_{1}<(d+2 \gamma) / s$. Then, the inequality

$$
\|f\|_{L_{k}^{p}\left(\mathbb{R}^{d}\right)} \leq C\left\|\left(-\triangle_{k}\right)^{s / 2} f\right\|_{L_{k}^{p_{1}}\left(\mathbb{R}^{d}\right)}^{\theta / p}\|f\|_{L_{k}^{p_{2}}\left(\mathbb{R}^{d}\right)}^{(p-\theta) / p}
$$

with

$$
\theta\left(\frac{1}{p_{1}}-\frac{s}{d+2 \gamma}\right)+\frac{p-\theta}{p_{2}}=1 .
$$

Theorem 37. Let $1<q<\infty, 0<s<d+2 \gamma$, and $1<p_{1}<(d+2 \gamma) / s$. Then, the inequality

$$
\begin{aligned}
& \exp \left(\left(\frac{1}{q}+\frac{s}{d+2 \gamma}-\frac{1}{p_{1}}\right)\right. \\
& \left.\quad \times \int_{\mathbb{R}^{d}} \frac{|f(x)|^{q}}{\|f\|_{L_{k}^{q}\left(\mathbb{R}^{d}\right)}^{q}} \ln \left(\frac{|f(x)|^{q}}{\|f\|_{L_{k}^{q}\left(\mathbb{R}^{d}\right)}^{q}}\right) \omega_{k}(x) d x\right) \\
& \leq C \frac{\left\|\left(-\triangle_{k}\right)^{s / 2} f\right\|_{L_{k}^{p_{1}}\left(\mathbb{R}^{d}\right)}}{\|f\|_{L_{k}^{q}\left(\mathbb{R}^{d}\right)}}
\end{aligned}
$$

holds for

$$
\frac{1}{q}+\frac{s}{d+2 \gamma}-\frac{1}{p_{1}}>0
$$

Corollary 38. Let $0<s<d+2 \gamma$ and $1<q<(d+2 \gamma) / s$, $f \in \dot{\mathscr{H}}_{q, k}^{s}\left(\mathbb{R}^{d}\right)$ such that $\|f\|_{L_{k}^{q}\left(\mathbb{R}^{d}\right)}=1$, one has

$$
\begin{aligned}
& \exp \left(\frac{s}{d+2 \gamma} \int_{\mathbb{R}^{d}}|f(x)|^{q} \ln \left(|f(x)|^{q}\right) \omega_{k}(x) d x\right) \\
& \leq C\left\|\left(-\triangle_{k}\right)^{s / 2} f\right\|_{L_{k}^{q}\left(\mathbb{R}^{d}\right)} .
\end{aligned}
$$

Theorem 39. One assumes that $G=\mathbb{Z}_{2}^{d}$. Let $1 \leq p<\infty$, $1 \leq p_{2}, q, q_{1}, q_{2}<\infty, 0<\theta<q, 0<s<d+2 \gamma$, and $1<p_{1}<(d+2 \gamma) / s$. Then, the inequality

$$
\|f\|_{L_{k}^{p, q}\left(\mathbb{R}^{d}\right)} \leq C\left\|\left(-\triangle_{k}\right)^{s / 2} f\right\|_{L_{k}^{p_{1}, q_{1}}\left(\mathbb{R}^{d}\right)}^{\theta / q}\|f\|_{L_{k}^{p_{2}, q_{2}}\left(\mathbb{R}^{d}\right)}^{(q-\theta) / q}
$$

holds for

$$
\begin{gathered}
\frac{\theta}{q_{1}}+\frac{q-\theta}{q_{2}}=1, \\
\theta\left(\frac{1}{p_{1}}-\frac{s}{d+2 \gamma}\right)+\frac{q-\theta}{p_{2}}=\frac{q}{p} .
\end{gathered}
$$

Remark 40. (i) We assume that $\mathrm{G}=\mathbb{Z}_{2}^{d}$. It follows from the special case $p_{1}=q_{1}$ and $p_{2}=q_{2}$ of (97) that the inequality

$$
\|f\|_{L_{k}^{p, q}\left(\mathbb{R}^{d}\right)} \leq C\left\|\left(-\triangle_{k}\right)^{s / 2} f\right\|_{L_{k}^{p_{1}}\left(\mathbb{R}^{d}\right)}^{\theta / q}\|f\|_{L_{k}^{p_{2}}\left(\mathbb{R}^{d}\right)}^{(q-\theta) / q}
$$

with $q=p(1-\theta s /(d+2 \gamma))$. Equation (99) can be thought of a refinement of (92) from (64).

(ii) We assume that $G=\mathbb{Z}_{2}^{d}$. It follows from the special case $p_{1}=q=\theta$ that (99) becomes

$$
\|f\|_{L_{k}^{q(d+2 \gamma) /(d+2 \gamma-q s), q}\left(\mathbb{R}^{d}\right)} \leq C\left\|\left(-\triangle_{k}\right)^{s / 2} f\right\|_{L_{k}^{q}\left(\mathbb{R}^{d}\right)},
$$

which can also be thought of as a refinement of the HardyLittlewood-Sobolev fractional integration theorem in Dunkl setting (cf. [21]):

$$
\left\|\left(-\triangle_{k}\right)^{-s / 2} f\right\|_{L_{k}^{q}\left(\mathbb{R}^{d}\right)} \leq C\|f\|_{L_{k}^{p}\left(\mathbb{R}^{d}\right)} .
$$

(iii) We note that the results of Dunkl-Riesz potential of this section are in sprit of the classical case (cf. [24]).

Theorem 41. One assumes that $G=\mathbb{Z}_{2}^{d}$. Let $1<p<\infty$, $0<s<(d+2 \gamma) / p$, and $1 \leq q \leq \infty$. There exists a positive constant $C$ such that one has

$$
\left\|\frac{f(x)}{\|x\|^{s}}\right\|_{L_{k}^{p, q}\left(\mathbb{R}^{d}\right)} \leq C\left\|\left(-\triangle_{k}\right)^{s / 2} f\right\|_{L_{k}^{p, q}\left(\mathbb{R}^{d}\right)} .
$$

For proof of this result, we need the following lemma which we prove as the Euclidean case.

Lemma 42. Let $1 \leq p_{1}, p_{2}, q_{1}, q_{2} \leq \infty$. If $f \in L_{k}^{p_{1}, q_{1}}\left(\mathbb{R}^{d}\right)$ and $g \in L_{k}^{p_{2}, q_{2}}\left(\mathbb{R}^{d}\right)$, then

$$
\|f g\|_{L_{k}^{p, q}\left(\mathbb{R}^{d}\right)} \leq C\|f\|_{L_{k}^{p_{1}, q_{1}}\left(\mathbb{R}^{d}\right)}\|g\|_{L_{k}^{p_{2}, q_{2}}\left(\mathbb{R}^{d}\right)},
$$

where $1 / p=1 / p_{1}+1 / p_{2}$ and $1 / q=1 / q_{1}+1 / q_{2}$.

Proof of Theorem 41. Let $1<p<\infty$ and $s \in(0,(d+2 \gamma) / p)$. We take $g(x)=1 /\|x\|^{s}$ and apply (103) in the specific form

$$
\|f g\|_{L_{k}^{p, q}\left(\mathbb{R}^{d}\right)} \leq C\|f\|_{L_{k}^{p_{1}, q}\left(\mathbb{R}^{d}\right)}\|g\|_{L_{k}^{r, \infty}\left(\mathbb{R}^{d}\right)},
$$

where $r=(d+2 \gamma) / s$ and $p_{1}=(q(d+2 \gamma)) /(d+2 \gamma-q s)$. As $g \in L_{k}^{r, \infty}\left(\mathbb{R}^{d}\right)$, we have

$$
\left\|\frac{f(x)}{\|x\|^{s}}\right\|_{L_{k}^{p, q}\left(\mathbb{R}^{d}\right)} \leq C\|f\|_{L_{k}^{((d+2 \gamma) p) /(d+2 \gamma-p s), q}\left(\mathbb{R}^{d}\right)},
$$

with $1 \leq q \leq \infty$. On the other hand, from [23], Theorem 1.2, we have

$$
\|f\|_{L_{k}^{((d+2 \gamma) p) /(d+2 \gamma-p s), q}\left(\mathbb{R}^{d}\right)} \leq C\left\|\left(-\triangle_{k}\right)^{s / s} f\right\|_{L_{k}^{p, q}\left(\mathbb{R}^{d}\right)},
$$

for any $f \in L_{k}^{p, q}\left(\mathbb{R}^{d}\right)$ with $1 \leq q \leq \infty, 1<p<\infty$, and $0<s<(d+2 \gamma) / p$. Thus, we obtain (102). 


\section{Dispersion Phenomena}

Notations. We denote by $\mathscr{I}_{k}(t)$ the Dunkl-Schrödinger semigroup on $L_{k}^{2}\left(\mathbb{R}^{d}\right)$ defined by

$$
\begin{aligned}
\mathscr{I}_{k}(t) v:= & \frac{1}{c_{k}|t|^{\gamma+d / 2}} e^{-i(d+2 \gamma)(\pi / 4) \operatorname{sgn} t} e^{i\left(\|\cdot\|^{2} / 4 t\right)} \\
& \times\left[\mathscr{F}_{D}\left(e^{i\left(\|\cdot\|^{2} / 4 t\right)} v\right)\right]\left(\frac{\cdot}{2 t}\right) .
\end{aligned}
$$

$W_{k}^{1, r}\left(\mathbb{R}^{d}\right)(1 \leq r \leq \infty)$ Banach space of (classes of) measurable functions $u: \mathbb{R}^{d} \rightarrow \mathbb{C}$ such that $T^{\mu} u \in L_{k}^{r}\left(R^{d}\right)$ in the sense of distributions, for every multi-index $\mu$ with $|\mu| \leq 1 . W_{k}^{1, r}\left(\mathbb{R}^{d}\right)$ is equipped with the norm

$$
\|u\|_{W_{k}^{1, r}\left(\mathbb{R}^{d}\right)}=\sum_{|\mu| \leq 1}\left\|T^{\mu} u\right\|_{L_{k}^{r}\left(\mathbb{R}^{d}\right)} .
$$

$W_{k, G}^{1, r}\left(\mathbb{R}^{d}\right)(1 \leq r \leq \infty)$ the subspace of $W_{k}^{1, r}\left(\mathbb{R}^{d}\right)$, which these elements are $G$-invariant.

Definition 43. One says that the exponent pair $(q, r)$ is $(d+2 \gamma) / 2$-admissible if $q, r \geq 2,(q, r,(d+2 \gamma) / 2) \neq(2, \infty, 1)$, and

$$
\frac{1}{q}+\frac{d+2 \gamma}{2 r} \leq \frac{d+2 \gamma}{4}
$$

If equality holds in (109) one says that $(q, r)$ is sharp $(d+2 \gamma) / 2$ admissible, otherwise, one says that $(q, r)$ is nonsharp $(d+$ $2 \gamma) / 2$-admissible. Note in particular that when $d+2 \gamma>2$, the endpoint

$$
P=\left(2, \frac{2 d+4 \gamma}{d+2 \gamma-2}\right)
$$

is sharp $(d+2 \gamma) / 2$-admissible.

Lemma 44 (see [25]). Let $E$ and $F$ be Banach spaces, and let $\mathscr{L}: L^{p, r}(0, \infty ; E) \rightarrow L^{q, s}(0, \infty ; F)$ be an integral operator for some $p, r, q, s$, with a kernel $k(t, \tau)$ such that

$$
\mathscr{L} f(t)=\int_{0}^{\infty} k(t, \tau) f(\tau) d \tau .
$$

If $1 \leq p \leq r<s \leq q<\infty$, then one has

$$
\|\widetilde{\mathscr{L}} f\|_{L^{q, s}(0, \infty ; F)} \leq C\|f\|_{L^{p, r}(0, \infty ; E)},
$$

where $\widetilde{\mathscr{L}}$ is the low diagonal operator defined by

$$
\widetilde{\mathscr{L}} f(t)=\int_{0}^{t} k(t, \tau) f(\tau) d \tau .
$$

Lemma 45. For any $(d+2 \gamma) / 2$-admissible pair $(q, r)$ with $q>2$

$$
\begin{gathered}
\left\|\mathscr{I}_{k}(t) f\right\|_{L^{q, 2}\left(0, \infty ; L_{k}^{r}\left(\mathbb{R}^{d}\right)\right)} \leq C\|f\|_{L_{k}^{2}\left(\mathbb{R}^{d}\right)}, \\
\left\|\int_{0}^{t} \mathscr{I}_{k}(t-\tau) g(\tau) d \tau\right\|_{L^{q, 2}\left(0, \infty ; L_{k}^{r}\left(\mathbb{R}^{d}\right)\right) \cap L^{\infty}\left(0, \infty ; L_{k}^{2}\left(\mathbb{R}^{d}\right)\right)} \\
\leq C\|g\|_{L^{q^{\prime}, 2}\left(0, \infty ; L_{k}^{r^{\prime}}\left(\mathbb{R}^{d}\right)\right)} .
\end{gathered}
$$

Proof. From the dispersion of $\mathscr{I}_{k}(t)$ such that

$$
\left\|\mathscr{I}_{k}(t) g\right\|_{L_{k}^{r}\left(\mathbb{R}^{d}\right)} \leq C t^{-(d+2 \gamma)(1 / 2-1 / r)}\|g\|_{L_{k}^{r^{\prime}}\left(\mathbb{R}^{d}\right)^{\prime}},
$$

for any $r \in[2, \infty],(\mathrm{cf} .[8])$, and the fact that

$$
\begin{aligned}
& t^{-(d+2 \gamma)(1 / 2-1 / r)} \in L^{2 r /(d+2 \gamma)(r-2), \infty}, \\
& \text { for any } r \in\left[2, \frac{2(d+2 \gamma)}{d+2 \gamma-2}\right],
\end{aligned}
$$

one can easily prove the result.

Theorem 46. Suppose that $d \geq 1,(q, r)$ and $\left(q_{1}, r_{1}\right)$ are $(d+2 \gamma) / 2$-admissible pairs and $2<a \leq q$. If $u$ is a solution to the problem

$$
\begin{gathered}
i \partial_{t} u(t, x)+\triangle_{k} u(t, x)=f(t, x), \quad(t, x) \in \mathbb{R} \times \mathbb{R}^{d}, \\
u_{\mid t=0}=u_{0},
\end{gathered}
$$

for some data, $u_{0}$, $f$, then

$$
\begin{aligned}
& \|u\|_{L^{q, a}\left(\mathbb{R} ; L_{k}^{r}\left(\mathbb{R}^{d}\right)\right)}+\|u\|_{C\left(\mathbb{R} ; L_{k}^{2}\left(\mathbb{R}^{d}\right)\right)} \\
& \leq C\left(\left\|u_{0}\right\|_{L_{k}^{2}\left(\mathbb{R}^{d}\right)}\right. \\
& \left.\quad+\|f\|_{L^{q_{1}^{\prime}, 2}\left(\mathbb{R} ; L_{k}^{r_{1}^{\prime}}\left(\mathbb{R}^{d}\right)\right) \cap L^{2}\left(\mathbb{R} ; L_{k}^{(2 d+4 y) /(d+2 \gamma+2), 2}\left(\mathbb{R}^{d}\right)\right)}\right) .
\end{aligned}
$$

Proof. Let $u$ be a solution of (118). We write $u$ as

$$
\begin{array}{r}
u(t, x)=\mathscr{I}_{k}(t) u_{0}(x)+\int_{0}^{t} \mathscr{I}_{k}(t-\tau) f(\tau, x) d \tau, \\
(t, x) \in \mathbb{R} \times \mathbb{R}^{d} .
\end{array}
$$

Let $k(t, \tau)=\mathscr{I}_{k}(t-\tau), E=L_{k}^{r_{1}^{\prime}}\left(\mathbb{R}^{d}\right)$ or $L_{k}^{(2 d+4 \gamma) /(d+2 \gamma+2), 2}\left(\mathbb{R}^{d}\right)$, $F=L_{k}^{r}\left(\mathbb{R}^{d}\right)$, and $\mathscr{L} f(t)=\int_{0}^{\infty} k(t, \tau) f(\tau) d \tau$. Then, since $q_{1}^{\prime} \leq 2<s \leq q$, in view of Lemma 44 , we only have to show that

$$
\begin{aligned}
& \left\|\int_{0}^{\infty} k(t, \tau) f(\tau) d \tau\right\|_{L^{q, s}\left(0, \infty ; L_{k}^{r}\left(\mathbb{R}^{d}\right)\right)} \\
& \quad \leq C\|f\|_{L^{q_{1}^{\prime}, 2}\left(0, \infty ; L_{k}^{r_{1}^{\prime}}\left(\mathbb{R}^{d}\right)\right) \cap L^{2}\left(0, \infty ; L_{k}^{(2 d+4 \gamma) /(d+2 \gamma+2), 2}\left(\mathbb{R}^{d}\right)\right)} .
\end{aligned}
$$

To show this, observe from (114) and $L^{q, s} \subset L^{q, 2}$ for all $s \geq 2$ that

$$
\begin{aligned}
& \left\|\int_{0}^{\infty} k(t, \tau) f(\tau) d \tau\right\|_{L^{q, s}\left(0, \infty ; L_{k}^{r}\left(\mathbb{R}^{d}\right)\right)}^{2} \\
& \quad \leq C \iint_{0}^{\infty}\left\langle\mathscr{I}_{k}(-\tau) f(\tau), \mathscr{I}_{k}(-y) f(y)\right\rangle d \tau d y .
\end{aligned}
$$

Then, from the endpoint result of Keel and Tao [26], the righthand side of (122) is bounded by $\|f\|_{L^{2}\left(0, \infty ; L_{k}^{(2 d+4 \gamma) /(d+2 \gamma+2), 2}\left(\mathbb{R}^{d}\right)\right)}^{2}$. The remaining part of theorem can be obtained by the duality of Lorentz space $\left(L^{q, s}\right)^{\prime}=L^{q^{\prime}, s^{\prime}}$ and the second part of (115). 
As an application of the previous theorem, we can derive Strichartz estimates of the solution to the following nonlinear problem:

$$
\begin{gathered}
i \partial_{t} u(t, x)+\triangle_{k} u(t, x) \\
=-|u(t, x)|^{4 /(d+2 \gamma-2)} u(t, x), \quad(t, x) \in \mathbb{R} \times \mathbb{R}^{d} \\
u_{\mid t=0}=u_{0} \in H_{k}^{1}\left(\mathbb{R}^{d}\right) \quad \text { in } \mathbb{R}^{d} .
\end{gathered}
$$

Theorem 47. If the initial data is sufficiently small and $G$-invariant, then there exists a unique solution $u \in$ $L^{q, s}\left(0, \infty ; W_{k, G}^{1, r}\left(\mathbb{R}^{d}\right)\right) \cap L^{2}\left(0, \infty ; W_{k, G}^{1,(2 d+4 \gamma) /(d+2 \gamma-2)}\left(\mathbb{R}^{d}\right)\right) \cap$ $C\left([0, \infty) ; H_{k, G}^{1}\left(\mathbb{R}^{d}\right)\right.$ for every sharp $(d+2 \gamma) / 2$-admissible pair $(q, r)$ with $q>2$ and $2<s \leq q$.

Proof. The existence of a unique $H_{k, G}^{1}\left(\mathbb{R}^{d}\right)$-solution is proved in [9], it suffices to prove that $u \in L^{q, s}\left(0, \infty ; W_{k, G}^{1, r}\left(\mathbb{R}^{d}\right)\right)$. From Duhamel's principle, we deduce that

$$
\begin{aligned}
u(t, x)= & \mathscr{I}_{k}(t) u_{0}(x) \\
& +\int_{0}^{t} \mathscr{I}_{k}(t-\tau)\left(|u(\tau, x)|^{4 /(d+2 \gamma-2)} u(\tau, x)\right) d \tau
\end{aligned}
$$

Using (114) and (119), we have

$$
\begin{aligned}
& \|T u\|_{L^{q, s}\left(\mathbb{R} ; L_{k}^{r}\left(\mathbb{R}^{d}\right)\right)} \\
& \quad \leq C\left(\left\|T u_{0}\right\|_{L_{k}^{2}\left(\mathbb{R}^{d}\right)}+\left\|T\left(|u|^{4 /(d+2 \gamma-2)} u\right)\right\|_{\left.L^{q_{1}^{\prime}, 2\left(\mathbb{R} ; L_{k}^{r^{\prime} 1}\left(\mathbb{R}^{d}\right)\right)}\right)}\right) .
\end{aligned}
$$

We can always find an admissible pair $\left(q_{0}, r_{0}\right)$ with $r_{0}<d+2 \gamma$ and $2<s_{0}<q_{0}$ and $\left(q_{1}, r_{1}\right)$ and $1<s_{1}<2$ such that

$$
\begin{gathered}
\frac{1}{q_{1}}=\frac{4}{(d+2 \gamma-2) q_{0}}+\frac{1}{q_{0}}, \quad \frac{1}{r_{1}}=\frac{4}{(d+2 \gamma-2) r_{1}}+\frac{1}{r_{0}} \\
\frac{1}{s_{1}}=\frac{4}{(d+2 \gamma-2) s_{0}}+\frac{1}{s_{0}}
\end{gathered}
$$

where $r^{*}=\left((d+2 \gamma) r_{0}\right) /\left(d+2 \gamma-r_{0}\right)$. Thus, from the Leibnitz rule, Hölder's inequality on Lorentz space, and Sobolev embedding, we deduce that

$$
\begin{aligned}
& \|T u\|_{L^{q_{0}, s_{0}}\left(\mathbb{R} ; L_{k}^{r_{0}}\left(\mathbb{R}^{d}\right)\right)} \\
& \quad \leq C\left(\left\|T u_{0}\right\|_{L_{k}^{2}\left(\mathbb{R}^{d}\right)}+\|T u\|_{L^{q_{0}, s_{0}}\left(\mathbb{R} ; L_{k}^{r_{0}}\left(\mathbb{R}^{d}\right)\right)}^{(d+2 \gamma+2) /(d+2 \gamma-2)}\right) .
\end{aligned}
$$

Since $\left\|u_{0}\right\|_{H_{k}^{1}\left(\mathbb{R}^{d}\right)}$ is small, we have

$$
\|T u\|_{L^{q_{0}, s_{0}}\left(\mathbb{R} ; L_{k}^{r_{0}}\left(\mathbb{R}^{d}\right)\right)} \leq C\left\|T u_{0}\right\|_{L_{k}^{2}\left(\mathbb{R}^{d}\right)} .
$$

Finally, since we can choose $\left(q_{1}, r_{1}\right)$ arbitrarily to be $(d+2 \gamma) / 2$ -admissible, for any $(d+2 \gamma) / 2$-admissible pair $(q, r)$ and $s$ with $q>2$ and $2<s \leq q$, we have

$$
\begin{aligned}
& \|T u\|_{L^{q, s}\left(\mathbb{R} ; L_{k}^{r}\left(\mathbb{R}^{d}\right)\right)} \\
& \quad \leq C\left(\left\|T u_{0}\right\|_{L_{k}^{2}\left(\mathbb{R}^{d}\right)}+\|T u\|_{L^{q_{0}, s_{0}}\left(\mathbb{R} ; L_{k}^{r_{0}}\left(\mathbb{R}^{d}\right)\right)}^{(d+2 \gamma+2) /(d+2 \gamma-2)}\right) \\
& \quad \leq C\left(\left\|T u_{0}\right\|_{L_{k}^{2}\left(\mathbb{R}^{d}\right)}+\left\|T u_{0}\right\|_{L_{k}^{2}\left(\mathbb{R}^{d}\right)}^{(d+2 \gamma+2) /(d+2 \gamma-2)}\right) .
\end{aligned}
$$

In a similar way, we can also derive from the smallness of $\left\|u_{0}\right\|_{H_{k}^{1}\left(\mathbb{R}^{d}\right)}$

$$
\|u\|_{L^{q, s}\left(\mathbb{R} ; L_{k}^{r}\left(\mathbb{R}^{d}\right)\right)} \leq C\left\|u_{0}\right\|_{L_{k}^{2}\left(\mathbb{R}^{d}\right)} .
$$

\section{Embedding Sobolev Theorems and Applications}

Theorem 48. Let $s, t>0, q_{1}, q_{2} \in[1, \infty]$ with $q_{1} \neq q_{2}$. Let $\theta=s /(s+t) \in(0,1), 1 / p=(1-\theta) / q_{1}+\theta / q_{2}$, and $r \in[1, \infty]$. If $f \in \dot{\mathscr{B}}_{q_{1}, r}^{s, k}\left(\mathbb{R}^{d}\right) \cap \dot{\mathscr{B}}_{q_{2}, r}^{-t, k}\left(\mathbb{R}^{d}\right)$, then $f \in L_{k}^{p, r}\left(\mathbb{R}^{d}\right)$, and one has

$$
\|f\|_{L_{k}^{p, r}\left(\mathbb{R}^{d}\right)} \leq C\|f\|_{\mathscr{\mathscr { B }}_{q_{1}, r}^{s, k}\left(\mathbb{R}^{d}\right)}^{1-\theta}\|f\|_{\mathscr{\mathscr { B }}_{q_{2}, r}^{-t, k}\left(\mathbb{R}^{d}\right)}^{\theta} .
$$

Proof. We start picking $p_{1}, p_{2}$ such that $1 \leq q_{1}<p_{1}<$ $p<p_{2}<q_{2} \leq \infty$ with $2 / p=1 / p_{1}+1 / p_{2}$. We have then $1 / p_{i}=\left(1-a_{i}\right) / q_{1}+a_{i} / q_{2}$ with $a_{i} \in(0,1)$ and $i=1,2$. We write

$$
\left\|\Delta_{j} f\right\|_{L_{k}^{p_{i}}\left(\mathbb{R}^{d}\right)} \leq\left\|\Delta_{j} f\right\|_{L_{k}^{q_{1}}\left(\mathbb{R}^{d}\right)}^{1-a_{i}}\left\|\Delta_{j} f\right\|_{L_{k}^{q_{2}}\left(\mathbb{R}^{d}\right)}^{a_{i}} .
$$

Using Hölder's inequality and by simple calculations, we obtain

$$
\begin{aligned}
& \sum_{j \in \mathbb{Z}} \varrho^{-j r / 2}\left\|\Delta_{j} f\right\|_{L_{k}^{p_{1}}\left(\mathbb{R}^{d}\right)}^{r} \leq\|f\|_{\dot{\mathscr{B}}_{q_{1}, r}^{s, k}\left(\mathbb{R}^{d}\right)}^{\left(1-a_{1}\right) r}\|f\|_{\dot{\mathscr{B}}_{q_{2}, r}^{-t, k}\left(\mathbb{R}^{d}\right)}^{r a_{1}}, \\
& \sum_{j \in \mathbb{Z}} \varrho^{j r / 2}\left\|\Delta_{j} f\right\|_{L_{k}^{p_{2}\left(\mathbb{R}^{d}\right)}}^{r} \leq\|f\|_{\dot{\mathscr{B}}_{q_{1}, r}^{s, k}\left(\mathbb{R}^{d}\right)}^{\left(1-a_{2}\right) r}\|f\|_{\dot{\mathscr{B}}_{q_{2}, r}^{-t, k}\left(\mathbb{R}^{d}\right)}^{r a_{2}},
\end{aligned}
$$

where $\varrho=2^{-2\left(s\left(1-a_{1}\right)-t a_{1}\right)}>0$. From this, and applying Proposition 25, we deduce that if $f \in \dot{\mathscr{B}}_{q_{1}, r}^{s, k}\left(\mathbb{R}^{d}\right) \cap \dot{\mathscr{B}}_{q_{2}, r}^{-t, k}\left(\mathbb{R}^{d}\right)$, then $f \in\left[L_{k}^{p_{1}}\left(\mathbb{R}^{d}\right), L_{k}^{p_{2}}\left(\mathbb{R}^{d}\right)\right]_{1 / 2, r}=L_{k}^{p, r}\left(\mathbb{R}^{d}\right)$. Furthermore, using (57), we finally have:

$$
\|f\|_{L_{k}^{p, r}\left(\mathbb{R}^{d}\right)} \leq C\|f\|_{\dot{\mathscr{B}}_{q_{1}, r}^{s, k}\left(\mathbb{R}^{d}\right)}^{1-\theta}\|f\|_{\dot{\mathscr{B}}_{q_{2}, r}^{-t, k}\left(\mathbb{R}^{d}\right)}^{\theta} .
$$

Corollary 49. Let $s$ be a real number in the interval $(0,(d+2 \gamma) / q)$, and let $q$ be a real number in $[1, \infty]$. There is a constant $C$ such that, for any function $f \in \dot{\mathscr{B}}_{q, q}^{s, k}\left(\mathbb{R}^{d}\right)$, the following inequality holds:

$$
\left(\int_{\mathbb{R}^{d}} \frac{|f(x)|^{q}}{\|x\|^{s q}} \omega_{k}(x) d x\right)^{1 / q} \leq C\|f\|_{\dot{\mathscr{B}}_{q, q}^{s, k}\left(\mathbb{R}^{d}\right)}^{\theta}\|f\|_{\dot{\mathscr{B}}_{\infty, q}^{s-(d+2 \gamma) / q, k}\left(\mathbb{R}^{d}\right)}^{1-\theta},
$$

where $\theta=1-q s /(d+2 \gamma)$. 
Proof. Let $p \in(1, \infty)$ and $s \in(0,(d+2 \gamma) / q)$ with $1 / p=1 / q-s /(d+2 \gamma)$. We take $g(x)=1 /\|x\|^{s}$ and apply (103) in the specific form

$$
\|f g\|_{L_{k}^{q, q}\left(\mathbb{R}^{d}\right)} \leq C\|f\|_{L_{k}^{p, q}\left(\mathbb{R}^{d}\right)}\|g\|_{L_{k}^{r, \infty}\left(\mathbb{R}^{d}\right)},
$$

where $r=(d+2 \gamma) / s$ and $p=(q(d+2 \gamma)) /(d+2 \gamma-q s)$. As $g \in L_{k}^{r, \infty}\left(\mathbb{R}^{d}\right)$, we have

$$
\left(\int_{\mathbb{R}^{d}} \frac{|f(x)|^{q}}{\|x\|^{s q}} \omega_{k}(x) d x\right)^{1 / q} \leq C\|f\|_{L_{k}^{p, q}\left(\mathbb{R}^{d}\right)} .
$$

Combining this with (131), we obtain (135).

Theorem 50. Let $0<s<(d+2 \gamma) / 2$ be given. There exists a positive constant $C$ such that for all function $u \in \dot{\mathscr{H}}_{2, k}^{s}\left(\mathbb{R}^{d}\right)$, one has

$$
\int_{\mathbb{R}^{d}} \frac{|u(x)|^{2}}{\|x\|^{2 s}} \omega_{k}(x) d x \leq C\|u\|_{\dot{\mathscr{H}}_{2, k}^{s}}^{2}\left(\mathbb{R}^{d}\right) .
$$

For proof of this theorem, we need the following lemma, which we obtain by simple calculations.

Lemma 51. Let $s$ be a real number in the interval $(0, \gamma+d / 2)$. Then, the function $x \mapsto\|x\|^{-2 s}$ belongs to the Dunkl-Besov space $\dot{\mathscr{B}}_{1, \infty}^{d+2 \gamma-2 s, k}\left(\mathbb{R}^{d}\right)$.

Proof of Theorem 50. Let us define

$$
I_{s, k}(u):=\int_{\mathbb{R}^{d}} \frac{|u(x)|^{2}}{\|x\|^{2 s}} \omega_{k}(x) d x=\left\langle\|\cdot\|^{-2 s}, u^{2}\right\rangle .
$$

Using homogeneous Littlewood-Paley decomposition and the fact that $u^{2}$ belongs to $\delta_{h, k}^{\prime}\left(\mathbb{R}^{d}\right)$, we can write

$$
\begin{aligned}
I_{s, k}(u)= & \sum_{|n-m| \leq 2}\left\langle\Delta_{n}\left(\|\cdot\|^{-2 s}\right), \Delta_{m}\left(u^{2}\right)\right\rangle \\
\leq C \sum_{|n-m| \leq 2}\left\langle 2^{n((d+2 \gamma) / 2-2 s)}\right. & \\
& \left.\quad \times \Delta_{n}\left(\|\cdot\|^{-2 s}\right), 2^{-m((d+2 \gamma) / 2-2 s)} \Delta_{m}\left(u^{2}\right)\right\rangle .
\end{aligned}
$$

Lemma 51 claims that $\|\cdot\|^{-2 s}$ belongs to $\dot{\mathscr{B}}_{2, \infty}^{(d+2 \gamma) / 2-2 s, k}\left(\mathbb{R}^{d}\right)$. Theorem 17 yields

$$
\left\|u^{2}\right\|_{\dot{\mathscr{B}}_{2,1}^{2 s-(d+2 \gamma) / 2, k}\left(\mathbb{R}^{d}\right)} \leq C\|u\|_{\dot{\mathscr{H}}_{2, k}^{s}\left(\mathbb{R}^{d}\right)}^{2} .
$$

Thus,

$$
I_{s, k}(u) \leq C\|u\|_{\dot{\mathscr{H}}_{2, k}^{s}\left(\mathbb{R}^{d}\right)}^{2} .
$$

The following results of this section are in sprit of the classical case (cf. [27]).
Theorem 52. Let $s, t>0, \theta=s /(s+t)$ and let $q_{1}, q_{2}, r_{1}, r_{2} \in$ $[1, \infty], p, r_{0} \in[1, \infty)$ with $1 / p=(1-\theta) / q_{1}+\theta / q_{2}$, $1 / r_{0}=(1-\theta) / r_{1}+\theta / r_{2}$.

(i) For every $f \in \dot{\mathscr{B}}_{q_{1}, r_{1}}^{s, k}\left(\mathbb{R}^{d}\right) \cap \dot{\mathscr{B}}_{q_{2}, r_{2}}^{-t, k}\left(\mathbb{R}^{d}\right)$, and if $r>r_{0}$, one has $f \in L_{k}^{p, r}\left(\mathbb{R}^{d}\right)$ and

$$
\|f\|_{L_{k}^{p, r}\left(\mathbb{R}^{d}\right)} \leq C\|f\|_{\dot{\mathscr{B}}_{q_{1}, r_{1}}^{s, k}}^{1-\theta}\left(\mathbb{R}^{d}\right)\|f\|_{\dot{\mathscr{B}}_{q_{2}, r_{2}}^{-t, k}\left(\mathbb{R}^{d}\right)}^{\theta} .
$$

(ii) Moreover, this inequality is valid for $r=r_{0}$ in the following cases:
(a) $r=r_{1}=r_{2}$,
(b) $r_{1}=q_{1}$ and $r_{2}=q_{2}$,
(c) $1<p \leq 2$ and $r_{0}=p$.

(iii) Finally, the condition $r \geq r_{0}$ is sharp.

Proof. (i) Case $r>r_{0}$. With no loss of generality, we may assume that $q_{1}<q_{2}$, and we fix $\varepsilon>0$ such that

$$
\begin{aligned}
\frac{1}{q_{2}}<\frac{1}{p}-\varepsilon\left(\frac{1}{q_{1}}-\frac{1}{q_{2}}\right) & =\frac{1}{p_{2}}<\frac{1}{p}+\varepsilon\left(\frac{1}{q_{1}}-\frac{1}{q_{2}}\right) \\
& =\frac{1}{p_{1}}<\frac{1}{q_{1}} .
\end{aligned}
$$

The proof follows essentially the same ideas used in the previous theorem. Indeed, we have for $M_{j}=2^{j s}\left\|\Delta_{j} f\right\|_{L_{k}^{q_{1}}\left(\mathbb{R}^{d}\right)}$ and $N_{j}=2^{-j t}\left\|\Delta_{j} f\right\|_{L_{k}^{q_{2}}\left(\mathbb{R}^{d}\right)}$ and for $\varepsilon_{0}=1$ and $\varepsilon_{1}=-1$,

$$
\begin{aligned}
\left\|\Delta_{j} f\right\|_{L_{k}^{p_{i}}\left(\mathbb{R}^{d}\right)} & \leq\left\|\Delta_{j} f\right\|_{L_{k}^{q_{1}}\left(\mathbb{R}^{d}\right)}^{1-\theta+\varepsilon \varepsilon_{i}}\left\|\Delta_{j} f\right\|_{L_{k}^{q_{2}}\left(\mathbb{R}^{d}\right)}^{\theta-\varepsilon \varepsilon_{i}} \\
& =M_{j}^{1-\theta+\varepsilon \varepsilon_{i}} N_{j}^{\theta-\varepsilon \varepsilon_{i}} 2^{-j \varepsilon \varepsilon_{i}(s+t)} .
\end{aligned}
$$

As $r_{1} \neq r_{2}$, we can only say that $\left(M_{j}^{1-\theta+\varepsilon \varepsilon_{i}} N_{j}^{\theta-\varepsilon \varepsilon_{i}}\right)_{j \in \mathbb{Z}} \in l^{\varrho_{i}}$, where $1 / \varrho_{i}=\left(1-\theta+\varepsilon \varepsilon_{i}\right) / r_{1}+\left(\theta-\varepsilon \varepsilon_{i}\right) / r_{2}$. We may use (57), but we get only that $f \in L_{k}^{p, \varrho}\left(\mathbb{R}^{d}\right)=\left[L_{k}^{p_{1}}\left(\mathbb{R}^{d}\right), L_{k}^{p_{2}}\left(\mathbb{R}^{d}\right)\right]_{1 / 2, \varrho}$ with $\varrho=\max \left(\varrho_{1}, \varrho_{2}\right)$ and that satisfies (143) with $r=\varrho$. However, we may choose $\varepsilon$ as small as we want and thus, $\varrho$ as close to $r_{0}$ as we want; thus $f$ satisfies (143) for every $r>r_{0}$.

(ii) Case $r=r_{0}$.

(a) If $r=r_{1}=r_{2}$ : this case was treated in Theorem 48 .

(b) If $r_{1}=q_{1}$ and $r_{2}=q_{2}$ : this is a direct consequence of (43), since we have

$$
\begin{aligned}
& \|f\|_{\dot{\mathscr{B}}_{q_{i}, q_{i}}^{s, k}\left(\mathbb{R}^{d}\right)}=\|f\|_{\dot{\mathscr{F}}_{q_{i}, q_{i}}^{s, k}\left(\mathbb{R}^{d}\right)} \leq C\|f\|_{\dot{\mathscr{F}}_{q_{i}, \infty}^{s, k}\left(\mathbb{R}^{d}\right)}, \\
& \|f\|_{\dot{\mathscr{B}}_{q_{i}, q_{i}}^{-t, k}\left(\mathbb{R}^{d}\right)}=\|f\|_{\dot{\mathscr{F}}_{q_{i}, q_{i}}^{-t, k}\left(\mathbb{R}^{d}\right)} \leq C\|f\|_{\dot{\mathscr{F}}_{q_{i}, \infty}^{-t, k}\left(\mathbb{R}^{d}\right)},
\end{aligned}
$$

we obtain

$$
\|f\|_{L_{k}^{p}\left(\mathbb{R}^{d}\right)} \leq C\|f\|_{\dot{\mathscr{B}}_{q_{1}, q_{1}}^{s, k}\left(\mathbb{R}^{d}\right)}^{1-\theta}\|f\|_{\dot{\mathscr{B}}_{q_{2}, q_{2}}^{-t, k}\left(\mathbb{R}^{d}\right)}^{\theta} .
$$


(c) Case $1<p \leq 2$ and $r_{0}=p$.

We just write

$$
\begin{aligned}
\left\|\Delta_{j} f\right\|_{L_{k}^{p}\left(\mathbb{R}^{d}\right)} & \leq\left\|\Delta_{j} f\right\|_{L_{k}^{q_{1}}\left(\mathbb{R}^{d}\right)}^{1-\theta}\left\|\Delta_{j} f\right\|_{L_{k}^{q_{2}}\left(\mathbb{R}^{d}\right)}^{\theta} \\
& =\left(2^{j s}\left\|\Delta_{j} f\right\|_{L_{k}^{q_{1}}\left(\mathbb{R}^{d}\right)}\right)^{1-\theta}\left(2^{-j t}\left\|\Delta_{j} f\right\|_{L_{k}^{q_{2}}\left(\mathbb{R}^{d}\right)}\right)^{\theta}
\end{aligned}
$$

and get by Hölder's inequality:

$$
\|f\|_{\dot{\mathscr{B}}_{p, p}^{0, k}\left(\mathbb{R}^{d}\right)} \leq C\|f\|_{\dot{\mathscr{B}}_{q_{1}, r_{1}}^{s, k}\left(\mathbb{R}^{d}\right)}^{1-\theta}\|f\|_{\dot{\mathscr{B}}_{q_{2}, r_{2}}^{-t, k}\left(\mathbb{R}^{d}\right)}^{\theta} .
$$

We then use the embedding $\dot{\mathscr{B}}_{p, p}^{0, k}\left(\mathbb{R}^{d}\right) \subset L_{k}^{p}\left(\mathbb{R}^{d}\right)=L_{k}^{p, p}\left(\mathbb{R}^{d}\right)$ which is valid for $p \leq 2$.

Theorem 53. Let $s, t>0$, let $q_{1}, q_{2} \in[1, \infty]$ with $q_{1}<q_{2}$. Let $\theta=s /(s+t) \in(0,1)$, and let $1 / p=(1-\theta) / q_{1}+\theta / q_{2}$.

(i) If $q_{1} \leq r_{1} \leq q_{2}$ and let $1 / r=(1-\theta) / r_{1}+\theta / q_{2}$. Then, one has

$$
\|f\|_{L_{k}^{p, r}\left(\mathbb{R}^{d}\right)} \leq C\|f\|_{\mathscr{\mathscr { q }}_{q_{1}, r_{1}}^{s, k}\left(\mathbb{R}^{d}\right)}^{1-\theta}\|f\|_{\dot{\mathscr{B}}_{q_{2}, q_{2}}^{-t, k}\left(\mathbb{R}^{d}\right)}^{\theta} .
$$

(ii) If $q_{1} \leq r_{2} \leq q_{2}$, and let $1 / r=(1-\theta) / q_{1}+\theta / r_{2}$. Then, one has

$$
\|f\|_{L_{k}^{p, r}\left(\mathbb{R}^{d}\right)} \leq C\|f\|_{\mathscr{S}_{q_{1}, q_{1}}^{s, k}\left(\mathbb{R}^{d}\right)}^{1-\theta}\|f\|_{\dot{\mathscr{B}}_{q_{2}, r_{2}}^{-t, k}\left(\mathbb{R}^{d}\right)}^{\theta} .
$$

Proof. We only prove the first inequality, as the proof for the second one is similar. Since $f \in \dot{\mathscr{B}}_{q_{1}, r_{1}}^{s, k}\left(\mathbb{R}^{d}\right)$, noting that $\lambda_{j}=2^{s j}\left\|\Delta_{j} f\right\|_{L_{k}^{q_{1}}\left(\mathbb{R}^{d}\right)}$, we have $\left(\lambda_{j}\right)_{j \in \mathbb{Z}} \in l^{r_{1}}$. Thus, using Proposition 26 (i) for the interpolation

$$
l^{r_{1}}=\left[l^{q_{1}}, l^{q_{2}}\right]_{a, r},
$$

with $1 / r_{1}=(1-a) / q_{1}+a / q_{2}$, we see that we have a partition $\mathbb{Z}=\sum_{j \in \mathbb{Z}} Z_{j}$ such that

$$
\begin{aligned}
& \left\|2^{-a j}\left(\sum_{n \in Z_{j}} \lambda_{n}^{q_{1}}\right)^{1 / q_{1}}\right\|_{l^{r_{1}}}+\left\|2^{(1-a) j}\left(\sum_{n \in Z_{j}} \lambda_{n}^{q_{2}}\right)^{1 / q_{2}}\right\|_{l^{r_{1}}} \\
& \quad \leq C\left\|\lambda_{j}\right\|_{l^{r_{1}}} .
\end{aligned}
$$

Moreover, since $f \in \dot{\mathscr{B}}_{q_{2}, q_{2}}^{-t, k}\left(\mathbb{R}^{d}\right)$, we have

$$
\left(\left(\sum_{j \in Z_{n}} 2^{-j q_{2} t}\left\|\Delta_{j} f\right\|_{L_{k}^{q_{2}}\left(\mathbb{R}^{d}\right)}^{q_{q_{2}}}\right)^{1 / q_{2}}\right)_{n \in \mathbb{Z}} \in l^{q_{2}} .
$$

Let us note that $M_{n}=\left(\sum_{j \in Z_{n}} 2^{-j q_{2} t}\left\|\Delta_{j} f\right\|_{L_{k}^{q_{2}}\left(\mathbb{R}^{d}\right)}^{q_{2}}\right)^{1 / q_{2}}, N_{n}=$ $2^{-a n}\left(\sum_{j \in Z_{n}} \lambda_{j}^{q_{1}}\right)^{1 / q_{1}}, L_{n}=2^{(1-a) n}\left(\sum_{j \in Z_{n}} \lambda_{j}^{q_{2}}\right)^{1 / q_{2}}$, and $f_{n}=$ $\sum_{j \in Z_{n}} \Delta_{j} f$. We apply now (147) and Theorem 48 to obtain

$$
\begin{aligned}
& \left\|f_{n}\right\|_{L_{k}^{p}\left(\mathbb{R}^{d}\right)} \leq C\left\|f_{n}\right\|_{\mathscr{S}_{q_{1}, q_{1}}^{s, k}}^{1-\theta}\left(\mathbb{R}^{d}\right)\left\|f_{n}\right\|_{\dot{\mathscr{S}}_{q_{2}, q_{2}}^{-t, k}\left(\mathbb{R}^{d}\right)}^{\theta} \\
& \leq C N_{n}^{1-\theta} M_{n}^{\theta} 2^{n a(1-\theta)}, \\
& \left\|f_{n}\right\|_{L_{k}^{p, q_{2}}\left(\mathbb{R}^{d}\right)} \leq C\left\|f_{n}\right\|_{\dot{\mathcal{B}}_{q_{1}, q_{2}}^{s, k}}^{1-\theta}\left(\mathbb{R}^{d}\right)\left\|f_{n}\right\|_{\dot{\mathscr{B}}_{q_{2}, q_{2}}^{-t, k}\left(\mathbb{R}^{d}\right)}^{\theta} \\
& \leq C L_{n}^{1-\theta} M_{n}^{\theta} 2^{-n(1-a)(1-\theta)} .
\end{aligned}
$$

Since we have $f=\sum_{n \in \mathbb{Z}} f_{n}$, with these two inequalities at hand, and using (57), we find that $f \in\left[L_{k}^{p}\left(\mathbb{R}^{d}\right), L_{k}^{p, q_{2}}\left(\mathbb{R}^{d}\right)\right]_{a, r}$, with $1 / r=(1-a) / p+a / q_{2}$, but, since $1 / r_{1}=(1-a) / q_{1}+a / q_{2}$ and $1 / p=(1-\theta) / q_{1}+\theta / q_{2}$, we obtain $\left[L_{k}^{p}\left(\mathbb{R}^{d}\right), L_{k}^{p, q_{2}}\left(\mathbb{R}^{d}\right)\right]_{a, r}=$ $L_{k}^{r}\left(\mathbb{R}^{d}\right)$ with $1 / r=(1-\theta) / r_{1}+\theta / q_{2}$.

Theorem 54. Let $s, t>0$, and let $q_{1}, q_{2} \in[1, \infty]$ with $q_{1}<q_{2}$. Let $\theta=s /(s+t) \in(0,1)$, and let $1 / p=(1-\theta) / q_{1}+\theta / q_{2}$. Let $q_{1} \leq r_{1} \leq r_{2} \leq q_{2}$, and let $1 / r=(1-\theta) / r_{1}+\theta / r_{2}$. Then, one has

$$
\left.\|f\|_{L_{k}^{p, r}\left(\mathbb{R}^{d}\right)} \leq C\|f\|_{\dot{\mathscr{B}}_{q_{1}, r_{1}}^{s, k}}^{1-\theta} \mathbb{R}^{d,}\right)\|f\|_{\dot{\mathscr{S}}_{q_{2}, r_{2}}^{-t, k}\left(\mathbb{R}^{d}\right)}^{\theta}
$$

Proof. Once the previous theorem is proved, it is enough to reapply similar arguments to obtain Theorem 54. As $q_{1}<r_{1}<r_{2}<q_{2}$, we start using

$$
l^{r_{1}}=\left[l^{q_{1}}, l^{r_{2}}\right]_{a, r_{1}}
$$

instead of (152), and we obtain a partition $\mathbb{Z}=\sum_{j \in \mathbb{Z}} Z_{j}$ such that

$$
\begin{aligned}
& \left\|2^{-a j}\left(\sum_{n \in Z_{j}} \lambda_{n}^{q_{1}}\right)^{1 / q_{1}}\right\|\left\|_{l^{r_{1}}}+\right\| 2^{(1-a) j}\left(\sum_{n \in Z_{j}} \lambda_{n}^{r_{2}}\right)^{1 / r_{2}} \|_{l^{r_{1}}} \\
& \leq C\left\|\lambda_{j}\right\|_{l^{r_{1}}},
\end{aligned}
$$

with $1 / r_{1}=(1-a) / q_{1}+a / r_{2}$ and where $\lambda_{j}=2^{s j}\left\|\Delta_{j} f\right\|_{L_{k}^{q_{1}}\left(\mathbb{R}^{d}\right)}$ belongs to $l^{r_{1}}$, since $f \in \dot{\mathscr{B}}_{q_{1}, r_{1}}^{s, k}\left(\mathbb{R}^{d}\right)$. Moreover, since $f \in$ $\dot{\mathscr{B}}_{q_{2}, r_{2}}^{-t, k}\left(\mathbb{R}^{d}\right)$, we have

$$
\left(\left(\sum_{j \in Z_{n}} 2^{-j q_{2} t}\left\|\Delta_{j} f\right\|_{L_{k}^{q_{2}}\left(\mathbb{R}^{d}\right)}^{q_{2}}\right)^{1 / q_{2}}\right)_{n \in \mathbb{Z}} \in l^{q_{2}} .
$$

Let us note that $M_{n}=\left(\sum_{j \in Z_{n}} 2^{-j q_{2} t}\left\|\Delta_{j} f\right\|_{L_{k}^{q_{2}}\left(\mathbb{R}^{d}\right)}^{q_{2}}\right)^{1 / q_{2}}, N_{n}=$ $2^{-a n}\left(\sum_{j \in Z_{n}} \lambda_{j}^{q_{1}}\right)^{1 / q_{1}}, L_{n}=2^{(1-a) n}\left(\sum_{j \in Z_{n}} \lambda_{j}^{q_{2}}\right)^{1 / q_{2}}$, and $f_{n}=$ $\sum_{j \in Z_{n}} \Delta_{j} f$. We apply now (151) and Theorem 48 instead of (155) to obtain

$$
\begin{aligned}
\left\|f_{n}\right\|_{L_{k}^{p, b}\left(\mathbb{R}^{d}\right)} & \left.\leq C\left\|f_{n}\right\|_{\dot{\mathscr{S}}_{q_{1}, q_{1}}^{s,}}^{1-\theta} \mathbb{R}^{d}\right)\left\|f_{n}\right\|_{\dot{\mathscr{S}}_{q_{2}, r_{2}}^{t+t,}\left(\mathbb{R}^{d}\right)}^{\theta} \\
& \leq C N_{n}^{1-\theta} M_{n}^{\theta} 2^{n a(1-\theta)},
\end{aligned}
$$


where $1 / b=(1-\theta) / q_{1}+\theta / r_{2}$ and

$$
\begin{aligned}
\left\|f_{n}\right\|_{L_{k}^{p, r_{2}}\left(\mathbb{R}^{d}\right)} & \leq C\left\|f_{n}\right\|_{\dot{\mathscr{B}}_{q_{1}, r_{2}}^{s, k}\left(\mathbb{R}^{d}\right)}^{1-\theta}\left\|f_{n}\right\|_{\dot{\mathscr{B}}_{q_{2}, r_{2}}^{-t, k}\left(\mathbb{R}^{d}\right)}^{\theta} \\
& \leq C N_{n}^{1-\theta} M_{n}^{\theta} 2^{-n(1-a)(1-\theta)} .
\end{aligned}
$$

Finally, we have via (57) that $f \in\left[L_{k}^{p, b}\left(\mathbb{R}^{d}\right), L_{k}^{p, r_{2}}\left(\mathbb{R}^{d}\right)\right]_{a, r}$, with $1 / r=(1-a) / b+a / r_{2}$. To conclude, we use the fact that $1 / b=$ $(1-\theta) / q_{1}+\theta / r_{2}$ and $1 / r_{1}=(1-a) / q_{1}+a / r_{2}$ in order to obtain that $f \in L_{k}^{p, r}\left(\mathbb{R}^{d}\right)$ with $1 / r=(1-\theta) / r_{1}+\theta / r_{2}$.

Conjecture 55. Theorems 34, 39, and 41 are true for the general reflection group $G$.

\section{Acknowledgments}

The author gratefully acknowledges the Deanship of Scientific Research at the University of Taibah University on material and moral support in the financing of this research Project No. 4001. The author is deeply indebted to the referees for providing constructive comments and for helping in improving the contents of this paper.

\section{References}

[1] C. F. Dunkl, "Differential-difference operators associated to reflection groups," Transactions of the American Mathematical Society, vol. 311, no. 1, pp. 167-183, 1989.

[2] T. H. Baker and P. J. Forrester, "Non symmetric Jack polynomials and integral kernels," Duke Mathematical Journal, vol. 95, no. 1, pp. 1-50, 1998.

[3] J. F. van Diejen and L. Vinet, Calogero-Sutherland-Moser Models, CRM Series in Mathematical Physics, Springer, New York, NY, USA, 2000.

[4] K. Hikami, "Dunkl operator formalism for quantum manybody problems associated with classical root systems," Journal of the Physical Society of Japan, vol. 65, no. 2, pp. 394-401, 1996.

[5] M. F. E. de Jeu, “The dunkl transform," Inventiones Mathematicae, vol. 113, no. 1, pp. 147-162, 1993.

[6] C. F. Dunkl, "Hankel transforms associated to finite reflection groups," Contemporary Mathematics, vol. 138, pp. 123-138, 1992.

[7] H. Mejjaoli, "Strichartz estimates for the Dunkl wave equation and application," Journal of Mathematical Analysis and Applications, vol. 346, no. 1, pp. 41-54, 2008.

[8] H. Mejjaoli, "Dispersion phenomena in Dunkl-Schrodinger equation and applications," Serdica Mathematical Journal, vol. 35, pp. 25-60, 2009.

[9] H. Mejjaoli, "Global well-posedness and scattering for a class of nonlinear Dunkl-Schrödinger equations," Nonlinear Analysis: Theory, Methods and Applications, vol. 72, no. 3-4, pp. 1121-1139, 2010.

[10] H. Mejjaoli, "Dunkl-heat semigroup and applications," Applicable Analysis, 2012.

[11] M. Rösler, "Generalized Hermite polynomials and the heat equation for Dunkl operators," Communications in Mathematical Physics, vol. 192, no. 3, pp. 519-542, 1998.

[12] T. Kawazoe and H. Mejjaoli, "Generalized Besov spaces and their applications," Tokyo Journal of Mathematics, vol. 35, no. 2, pp. 297-320, 2012.
[13] H. Mejjaoli, "Generalized homogeneous Besov spaces and their applications," Serdica Mathematical Journal, vol. 38, no. 4, pp. 575-614, 2012.

[14] C. F. Dunkl, "Integral kernels with re ection group invariant," Canadian Journal of Mathematics, vol. 43, pp. 1213-1227, 1991.

[15] M. Rösler, "A positive radial product formula for the Dunkl kernel," Transactions of the American Mathematical Society, vol. 355, no. 6, pp. 2413-2438, 2003.

[16] S. Thangavelu and Y. Xu, "Convolution operator and maximal function for the Dunkl transform," Journal d'Analyse Mathematique, vol. 97, pp. 25-55, 2005.

[17] K. Trimèche, "Paley-Wiener theorems for the Dunkl transform and Dunkl translation operators," Integral Transforms and Special Functions, vol. 13, no. 1, pp. 17-38, 2002.

[18] P. Etingof, "A uniform proof of the macdonald-Mehta-Opdam identity for finite coxeter groups," Mathematical Research Letters, vol. 17, no. 2, pp. 277-282, 2010.

[19] S. Thangavelyu and Y. Xu, "Riesz transform and Riesz potentials for Dunkl transform," Journal of Computational and Applied Mathematics, vol. 199, no. 1, pp. 181-195, 2007.

[20] J. Bergh and J. Löfström, Interpolation Spaces. An Introduction, Springer, New York, NY, USA, 1976.

[21] S. Hassani, S. Mustapha, and M. Sifi, "Riesz potentials and fractional maximal function for the dunkl transform," Journal of Lie Theory, vol. 19, no. 4, pp. 725-734, 2009.

[22] J. Merker, "Regularity of solutions to doubly nonlinear diffusion equations," Electronic Journal of Differential Equations, vol. 17, pp. 185-195, 2009.

[23] M. G. Hajibayov, "Boundedness of the Dunkl convolution operators," in Analele Universitatii de Vest, vol. 49 of Timisoara Seria Matematica Informatica, pp. 49-67, 2011.

[24] H. Hajaiej, X. Yu, and Z. Zhai, "Fractional Gagliardo-Nirenberg and Hardy inequalities under Lorentz norms," Journal of Mathematical Analysis and Applications, vol. 396, no. 2, pp. 569-577, 2012.

[25] C. Ahn and Y. Cho, "Lorentz space extension of Strichartz estimates," Proceedings of the American Mathematical Society, vol. 133, no. 12, pp. 3497-3503, 2005.

[26] M. Keel and T. Tao, "Endpoint Strichartz estimates," American Journal of Mathematics, vol. 120, no. 5, pp. 955-980, 1998.

[27] D. Chamorro and P. G. Lemarié-Rieusset, "Real Interpolation method,Lorentz spaces and refined Sobolev inequalities," http://arxiv.org/abs/1211.3320. 


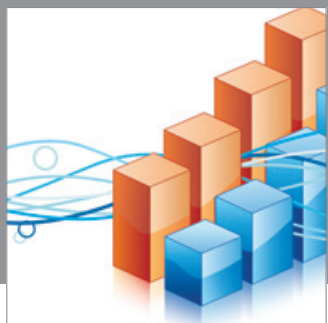

Advances in

Operations Research

mansans

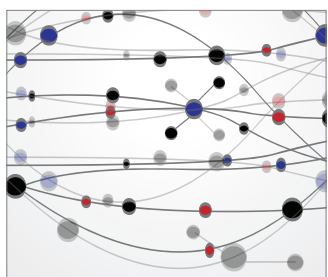

The Scientific World Journal
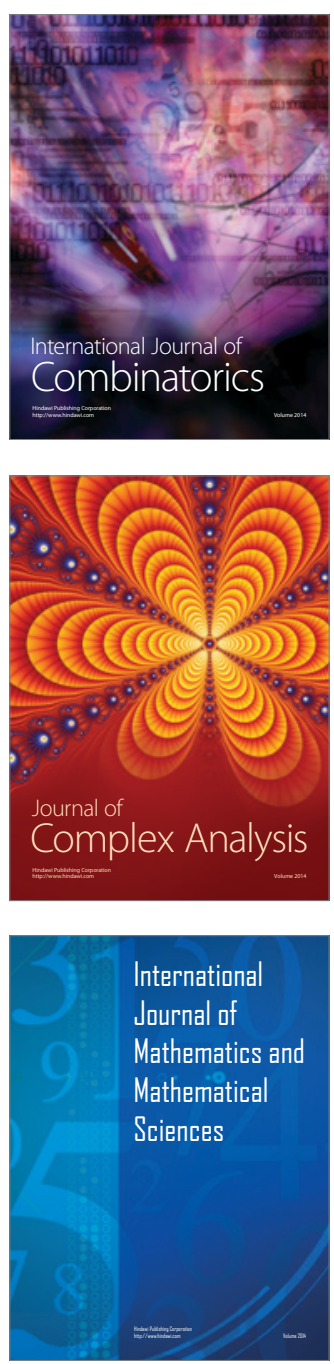
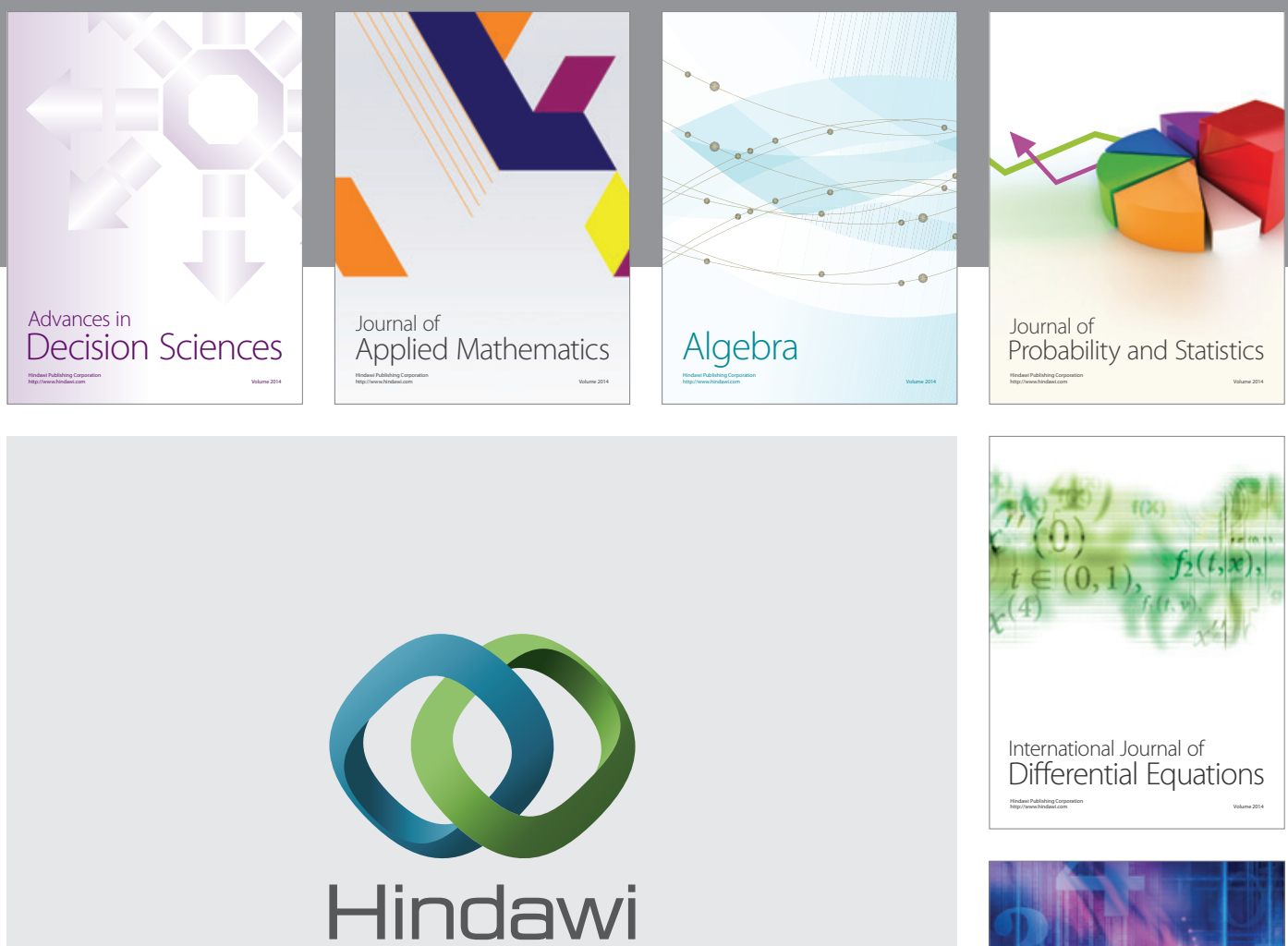

Submit your manuscripts at http://www.hindawi.com
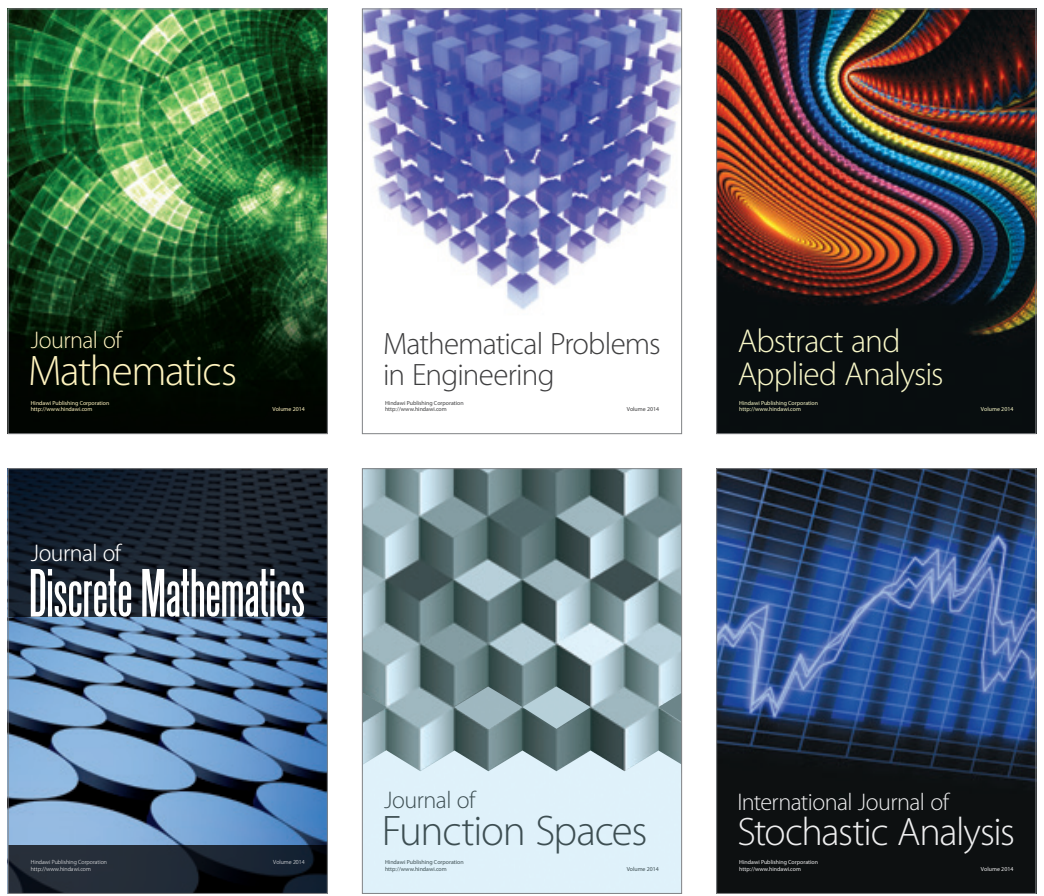

Journal of

Function Spaces

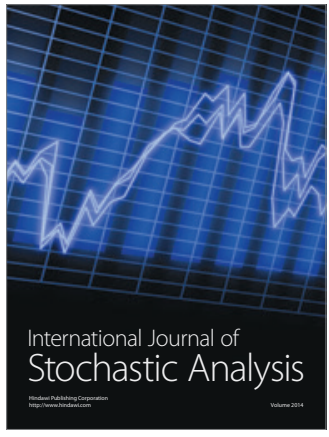

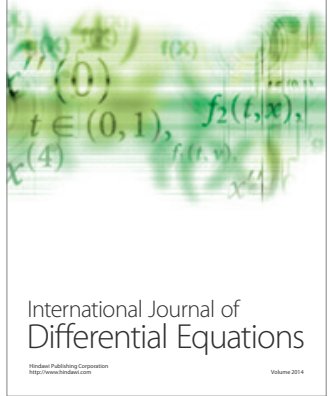
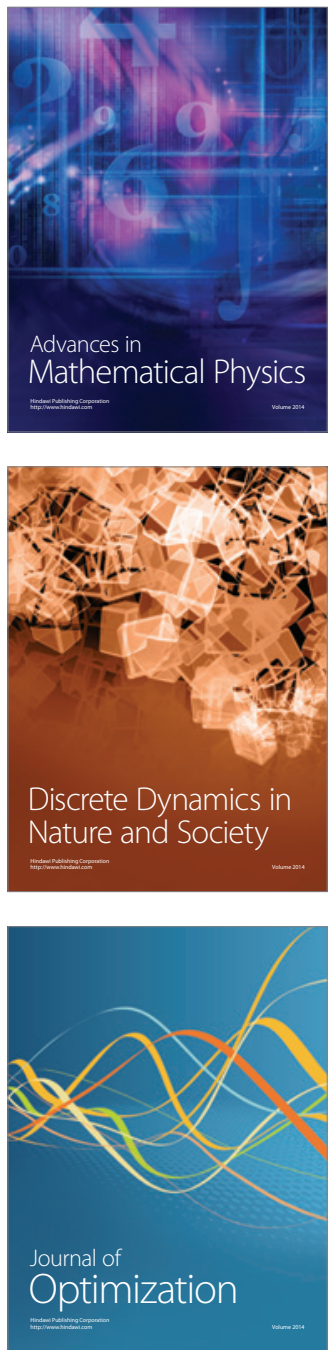